\title{
Distribución geográfica de Hylocereus (CActaceae) EN MÉXICO
}

\author{
luz Adriana García-Rubio ${ }^{1}$, Ofelia Vargas-Ponce ${ }^{1,3}$, Felipe de Jesús Ramírez-Mireles, \\ Guadalupe Munguía-Lino ${ }^{1}$, Carlos Alberto Corona-Oceguera ${ }^{1}$ y Tolin Cruz-Hernández ${ }^{2}$ \\ ${ }^{1}$ Universidad de Guadalajara, Zapopan, Jalisco, México. \\ ${ }^{2}$ Universidad Autónoma de Chapingo, Chapingo, Estado de México, México \\ ${ }^{3}$ Autora para correspondencia: vargasofelia@gmail.com
}

\begin{abstract}
Resumen: Hylocereus es un género americano de importancia hortícola por sus frutos comestibles. En México crecen varias especies de Hylocereus. Sin embargo, su distribución no se conoce por completo, por ello se requiere ampliar este conocimiento. Con este objetivo se analizaron la riqueza de especies y los patrones de distribución geográfica de Hylocereus en México. Se revisaron ejemplares de 12 herbarios nacionales y se hicieron colectas botánicas. En adición, con un Sistema de Información Geográfica y 437 registros georeferenciados se identificaron las provincias biogeográficas y condiciones ecológicas en que se desarrollan. Como resultado, se reconoció a $H$. ocamponis, $H$. purpusii, H. undatus e H. aff. escuintlensis. Jalisco, Chiapas y Oaxaca tienen el mayor número de registros y junto con Michoacán y Guerrero son los de mayor riqueza. Los cuatro taxa se desarrollan en la Costa Pacífica Mexicana mientras H. purpusii, H. ocamponis y H. undatus confluyen en el Eje Volcánico Transmexicano. Hylocereus aff. escuintlensis e H. undatus convergen en la provincia de Chiapas. Hylocereus crece en climas templados a muy cálidos; $H$. undatus exhibe la mayor amplitud ecológica, $H$. aff. escuintlensis muestra intervalos más estrechos mientras que H. ocamponis e H. purpusii coexisten en condiciones similares.
\end{abstract}

Palabras clave: biogeografía; México; pitahaya; riqueza; SIG.

\begin{abstract}
Hylocereus is an American genus with horticultural importance for its edible fruits. In Mexico several species of $\mathrm{Hy}$ locereus grow. However their distributions are not fully understood. Therefore, it is required to extend this knowledge. With this objective species richness and distribution geographical patterns were analyzed. Voucher specimens of twelve national herbaria were reviewed and field collections were made. Based on 437 georeferenced records and using a Geographic Information System, we identified the biogeographical provinces and ecological conditions the plants grow in. Hylocereus ocamponis, H. purpusii, $H$. undatus, and $H$. aff. escuintlensis were identified. Jalisco, Chiapas and Oaxaca had the largest number of records and along with Michoacán and Guerrero showed the greatest species richness. Further, the Mexican Pacific Coast was the richest biogeographical province, the four taxa were found there. Hylocereus purpusii, H. ocamponis and H. undatus converged at the Transmexican Volcanic Belt. Meanwhile, $H$. aff. escuntlensis and $H$. undatus occurred at the Chiapas province. Hylocereus grows in temperate, warm and very warm climates. Moreover, $H$. undatus exhibited the greatest ecological range, but $H$. aff. escuintlensis showed the narrowest, and Hylocereus ocamponis and H. purpusii coexist in similar ecological conditions.
\end{abstract}

Key words: biogeography; GIS; Mexico; pitahaya; species richness.

$H$ ylocereus Britton \& Rose (Cactaceae) es un género nativo de América de importancia económica, por sus frutos comestibles y su alta tolerancia a la sequía (Anderson, 2001; Tel-Zur et al., 2011). Agrupa 18 especies que se conocen comúnmente como pitahaya que crecen en áreas templadas, tropicales, subtropicales y semiáridas de México, Centro América y Norte de Sudamérica (Britton y Rose, 1963; Anderson, 2001). Se distingue por su forma de creci- miento trepador, tallos trialados, más o menos ondulados, con raíces aéreas y flores aromáticas de apertura nocturna (Bravo-Hollis, 1978). Uno de sus atributos más notorios son los frutos globosos con escamas foliáceas y exocarpo de color amarillo, rojo y magenta. El endocarpo es de color blanco, amarillo o magenta con numerosas semillas diminutas (Bravo-Hollis, 1978; Anderson, 2001). Los frutos han sido alimento recurrente para los pobladores locales y for- 
man parte de los productos que se ofrecen en los mercados tradicionales.

El uso tradicional de Hylocereus costaricensis Britton \& Rose, H. ocamponis (Salm-Dyck) Britton \& Rose, H. monacanthus (Lem.) Britton \& Rose [= H. polyrhizus (F.A.C. Weber) Britton \& Rose] e H. undatus (Haw.) Britton \& Rose, ha ocurrido por siglos en sus áreas de distribución natural como Colombia, Costa Rica, Guatemala, México, Nicaragua y Perú (Rodríguez-Canto, 2000; Hart, 2005; Le Bellec et al., 2006; Ortiz-Hernández y Carrillo-Salazar, 2012; Ortiz-Hernández et al., 2012). En las últimas tres décadas, el sabor, forma y color del fruto han abierto un nicho dentro del mercado comercial de frutos exóticos en Europa, Asia y América (Le Bellec et al., 2006; Ortiz-Hernández y Carrillo-Salazar, 2012). Esto ha propiciado el establecimiento de plantaciones comerciales con mayor tecnificación en los Estados Unidos de América, Israel, Malasia y Tailandia (Nerd et al., 2002; Le Bellec et al., 2006; Tel-Zur et al., 2011; Ortiz-Hernández y Carrillo-Salazar, 2012).

La importancia de Hylocereus como cultivo comercial ha estimulado el interés por alcanzar un mayor conocimiento del género. En las últimas dos décadas se han conducido diversos estudios a nivel mundial (Le Bellec, 2006; OrtizHernández y Carrillo-Salazar, 2012; Ortiz-Hernández et al., 2012). Un área de investigación que ha recibido mayor atención es la relacionada con el establecimiento del cultivo, sistemas de producción, fitosanidad, manejo poscosecha y comercialización (INTA 2014; Hoa et al., 2006; Masyahit et al., 2009; Awang et al., 2011: INTA, 2014; Mizrahi, 2014). También se ha evaluado la respuesta fisiológica y desarrollo de Hylocereus ante la disponibilidad de $\mathrm{CO}_{2}$, nutrientes (Nobel y De la Barrera, 2002; Ben-Asher et al., 2006), condiciones de sombra (Raveh et al., 1998), así como aspectos ambientales que tienen un efecto en el inicio de la floración (Khaimov y Mizrahi, 2006; Serna-Cock et al., 2011; Jiang et $a l ., 2012)$. Destacan los estudios sobre aspectos de genética, hibridación de clones y caracterización realizados en Israel, uno de los principales productores de pitahaya (Cisneros y Tel-Zur, 2010, 2013; Lichtenzveig et al., 2000; Tel-Zur et al., 2004, 2011).

En el ámbito nacional hay avances importantes en el estudio de Hylocereus, con mayor énfasis en $H$. undatus por ser una de las especies con buena aceptación en el mercado internacional así como por su importancia cultural. Se han realizado caracterización morfológica (Maldonado-Poot, 2000; Castillo-Martínez, 2002; Grimaldo-Juárez et al., 2007), fitosanitaria (Valencia-Botín et al., 2003; 2004) y genética de algunos individuos cultivados (Ramírez-Mireles, 1999; Castillo-Martínez et al., 2003; Legaria et al., 2005). Se ha estudiado su sistema reproductivo (Valiente-Banuet $e t$ al., 2007) y fisiología (Ortiz-Hernández et al., 1996; 1999), entre otros. Además, hay evidencia de la importancia cultural de esta especie y su uso como alimento y ornamental y en menor proporción como medicinal en varias áreas del país
(Ortiz-Hernández et al., 2012). En adición, se reconoce que el aprovechamiento ocurre en poblaciones silvestres, cultivadas en traspatio y cultivos tipo huerta con mayor densidad de individuos (Rodríguez-Canto, 2000; Valiente-Banuet et al., 2007; Ortiz-Hernández et al., 2012). En cambio para las otras especies de Hylocereus que crecen en el país estos aspectos son menos conocidos.

La taxonomía de Hylocereus es uno de los aspectos que no se han resuelto por completo. Se señala que el género es poco colectado y estudiado y algunas especies son pobremente conocidas (Bravo-Hollis y Arias, 2011). Cactaceae es monofilética (Nyffeler, 2002) al igual que la subfamilia Cactoideae (Terrazas y Arias, 2002) en la que se incluye la tribu Hylocereeae; sin embargo, esta tribu y otras ocho reconocidas por Barthlott y Hunt (1993) con base en datos morfológicos y anatómicos no son monofiléticas (Terrazas y Arias, 2002). El estudio sistemático de Hylocereus ha sido atendido por diversos autores. Britton y Rose $(1920,1963)$ reconocieron 18 especies y Backeberg (1976) enumeró 24. En estudios más recientes Hunt (1999) y Anderson (2001) reconocen 18 taxa, aunque más tarde Hunt (2006) acepta 14. En el caso particular de México, Bravo-Hollis (1978) reconoció tres especies, $H$. ocamponis, $H$. purpusii Britton $\&$ Rose e $H$. undatus y mencionó la posible existencia de otras cuatro $[H$. guatemalensis (Eichlam) Britton \& Rose, H. calcaratus Britton \& Rose, H. napoleonis Britton \& Rose e H. stenopterus Britton \& Rose] con base en materiales observados en campo y esta última especie cultivada en el Jardín Botánico de la UNAM; sin embargo, éstas no fueron corroboradas. Guzmán et al. (2003) adicionaron a la lista a H. minutiflorus Britton \& Rose mientras que Cálix de Dios (2004) señaló nueve especies (H. calcaratus, H. escuintlensis Kimnach, H. guatemalensis, H. ocamponis, H. purpusii, H. stenopterus, H. triangularis (L.) Britton \& Rose, H. trigonus Saff. e H. undatus,). Por su parte García-Aguilar (2007) a partir de una revisión de la historia sistemática de Hylocereus y el estudio de atributos morfológicos, anatómicos (García-Aguilar et al., 2009) y genéticos (García-Aguilar et $a l ., 2013)$ en diez poblaciones colectadas en el país reconoce para México a H. ocamponis, H. purpusii e H. undatus. Estos autores no reconocen seis de las especies que citó Calix de Dios (2004) y no hacen referencia a H. minutiflorus.

De igual forma, los antecedentes de estudios de distribución de Hylocereus en México son pocos. Cálix de Dios (2004) modeló la distribución y condiciones ecológicas en las que ocurre el género en México, utilizando el mapa de provincias florísticas de Cronquist (1982), la clasificación de los tipos de vegetación de México de Miranda y Hernández (1963) y la vegetación de México de Rzedowski (1978). Sin citar los especímenes de referencia y sin especificar la condición silvestre o cultivada de las muestras, este autor indica que Hylocereus crece en el sureste (Península de Yucatán, Meseta central de Chiapas), centro-occidente (Costa del Golfo y del Pacífico, Planicie Central de México, Re- 
gión del Bajío, la Costa del Occidente, el Altiplano Potosíno-Zacatecano), y en el norte de México en los estados de Sinaloa, Durango, Tamaulipas y San Luis Potosí. Sotelo-Ruiz et al. (2005) utilizaron un sistema de información geográfica (SIG) y variables edafológicas y bioclimáticas para modelar las zonas potenciales para el establecimiento de plantaciones de Hylocereus spp. en el sur del Estado de México. Posteriormente, García-Aguilar (2007) empleó un SIG y con base en diez colectas de campo que representan a $H$. ocamponis, $H$. purpusii e $H$. undatus indicó dos regiones importantes de distribución: la primera en Nayarit y Jalisco y la segunda en Guerrero. En este contexto, es importante completar el conocimiento de los patrones de distribución del género incrementando el número de especímenes. Por lo anterior, el objetivo de este trabajo fue analizar los patrones de distribución geográfica de Hylocereus en el país.

\section{Materiales y métodos}

Revisión de colecciones biológicas y colecta de Hylocereus. Se hizo una revisión de literatura que incluyó trabajos florísticos, taxonómicos y temas diversos relacionados al género. Además, se revisaron los ejemplares de 12 colecciones botánicas nacionales: el Herbario Hortorio del Colegio de Postgraduados, Montecillo, México (CHAPA), el Herbario del Centro de Investigación Científica de Yucatán (CICY), el Herbario del Centro Interdisciplinario de Investigación para el Desarrollo Integral Regional del Instituto Politécnico Nacional en Oaxaca (OAX), el Herbario Eizi Matuda de la Facultad de Ciencias Agrícolas de la Universidad Autónoma del Estado de México (CODAGEM), el Herbario de la Escuela Nacional de Ciencias Biológicas del Instituto Politécnico Nacional (ENCB), el Herbario de la Universidad de Ciencias y Artes de Chiapas (HEM), el Herbario de la Universidad Autónoma del Estado de Morelos (HUMO), el Herbario Luz María Villarreal de Puga del Instituto de Botánica de la Universidad de Guadalajara (IBUG), el Herbario del Centro Regional del Bajío (IEB) del Instituto de Ecología, A.C., el Herbario Nacional del Instituto de Biología de la Universidad Nacional Autónoma de México (MEXU), el Herbario del Instituto de Ecología, A.C., Xalapa (XAL) y el Herbario del Instituto Manantlán de ecología y biodiversidad del Centro Universitario de la Costa Sur de la Universidad de Guadalajara (ZEA). De cada ejemplar se registraron los datos de la etiqueta, se revisó su procedencia y se anotó su condición cultivada cuando se precisa que procede de un huerto, traspatio o jardín. Además se tomaron fotografías como apoyo para ratificar la determinación taxonómica de los ejemplares de herbario revisados. La determinación se hizo utilizando la metodología clásica, se usaron claves de identificación, se revisaron las descripciones botánicas descritas por diversos autores (Britton y Rose, 1963; Kimnach, 1984; Anderson, 2001; Bravo-Hollis y Arias, 2011; Véliz Pérez, 2011; Véliz Pérez y Arias, 2013). En adición, se con- sultaron fotografías de especímenes (isotipos) de H. escuintlensis disponibles en línea (Kew Royal Botanical Gardens (K); Smithsonian National Museum of Natural History (US); The Field Museum (F) y la Universidad Autónoma de México (UNIBIO colecciones biológicas).

El trabajo de campo incluyó la exploración y la colecta de Hylocereus en Jalisco, Chiapas, Michoacán, Nayarit, Sinaloa, Guanajuato, Querétaro y Oaxaca durante los años 2009 a 2013. Se obtuvieron tallos de 219 localidades y se tomaron fotografías. Para cada ejemplar colectado se hizo la determinación taxonómica con los mismos criterios que se utilizaron para los ejemplares de herbario. Dos tallos de cada localidad se depositaron en el banco de germoplasma del SINAREFI en Coatepec de Harinas, Estado de México. En adición 139 ejemplares se conservan en cultivo como duplicados en el Jardín Botánico del Instituto de Botánica de la Universidad de Guadalajara y 80 ejemplares también cultivados se conservan en Puebla bajo resguardo de la Universidad Autónoma Chapingo. Además, se prepararon ejemplares botánicos de las 219 muestras y se depositaron en los herbarios IBUG y MEXU. En este estudio no se incluyeron los ejemplares cultivados como ornamentales en jardines de ciudades, debido a la dificultad del muestreo y registro; las muestras observadas en esta condición corresponden a $H$. undatus.

Análisis de distribución. El análisis de distribución se hizo mediante una base de datos con 437 registros, que contienen la información registrada en las etiquetas de los ejemplares de herbario y los colectados en este trabajo. Los campos de la base abarcaron datos taxonómicos, curatoriales, geográficos y ecológicos. Los datos taxonómicos incluyeron: familia, género, epíteto específico, autor, nombre del colector, número colecta. Los curatoriales consideraron: el nombre de colectores, número de colecta, el determinador y la colección donde está depositado. Los datos geográficos refirieron al país, estado, municipio, localidad, altitud, latitud y longitud utilizando coordenadas geográficas. Los datos ecológicos incluyeron el tipo de vegetación, especies asociadas, observaciones y fecha de colecta.

Los registros que carecían de coordenadas (grados, minutos y segundos o grados decimales) y altitud (m s.n.m.) fueron georeferenciados empleando la base de datos de localidades de México de la Comisión Nacional para el Conocimiento y Uso de la Biodiversidad (CONABIO) y Google Earth (Google Inc., disponible en en linea en <www. earth.google.com>, consultado entre marzo y noviembre de 2013). La base de datos se depuró eliminando registros repetidos y se corrigieron aquellos con georreferencias erróneas. Finalmente, la base de datos fue transformada a un formato compatible con un Sistema de Información Geográfica (SIG). En el programa ArcView GIS 3.3 (ESRI, 1992-2002) se visualizaron los puntos de distribución de Hylocereus y se elaboraron mapas de distribución utilizando 
exclusivamente los registros de especímenes silvestres por división política, provincias biogeográficas de México, tipo de vegetación e isotermas medias anuales. Para determinar las condiciones ecológicas en las que crece Hylocereus en el país, se sobrepusieron a los puntos de distribución los mapas de provincias biogeográficas de México (Morrone, 2005), vegetación potencial (Rzedowski, 1990), isotermas medias anuales (García-E.-CONABIO, 1998), precipitación media anual (Vidal-Zepeda, 1990) y cuencas hidrográficas de México (INEGI-INE-CONAGUA, 2007) y se realizó el geoprocesamiento de datos.

\section{Resultados}

A partir de la determinación taxonómica de los especímenes de herbario y los recolectados en campo se reconocieron cuatro especies de Hylocereus para México: H. ocamponis, $H$. purpusii, $H$. undatus e $H$. aff. escuintlensis. Sólo ocho especímenes morfológicamente similares que fueron colectados en Oaxaca no pudieron ser identificados y se dejaron como Hylocereus sp. La base de datos generada para el análisis integró 437 registros únicos (Apéndice 1). Ésta se conformó de 218 especímenes de herbario y 219 colectados.
Hylocereus undatus fue la especie mejor representada con 247 colectas, le siguieron $H$. purpusii con $116, H$. ocamponis con 55, $H$. aff. escuintlensis con 12 e Hylocereus sp. con ocho. Jalisco, Chiapas y Oaxaca fueron los estados con el mayor número de registros $(72,59$ y 55 respectivamente). Del total de los registros, 270 fueron de plantas silvestres, 146 cultivadas y 21 especímenes de herbario carecen de esta información, por lo que estos últimos no se contabilizaron en ninguna de las dos condiciones señaladas. El número de registros por estado dentro de la condición silvestre y cultivada se observan en la tabla 1. Los estados con mayor riqueza de especies silvestres fueron Guerrero, Jalisco y Michoacán donde crecen $H$. ocamponis, $H$. purpusii e $H$. undatus, al igual que Oaxaca en donde habitan $H$. purpusii, H. undatus y los especímenes catalogados como Hylocereus sp. En Campeche y Yucatán se registró sólo a $H$. undatus y en Nayarit y Sinaloa a $H$. purpusii. En el caso de $H$. aff. escuintlensis únicamente se ubicó en Chiapas.

En México las especies de Hylocereus se ubican entre $\operatorname{los} 15^{\circ} 16^{\prime}$ y $25^{\circ} 3^{\prime} \mathrm{N}, 89^{\circ} 27^{\prime}$ y $107^{\circ} 25^{\prime} \mathrm{O}$. Su distribución natural incluye 21 estados, el más norteño es Sinaloa y el más sureño Quintana Roo; abarca la vertiente pacífica y porciones tropicales del Golfo, pasando por el Occidente,

Tabla 1. Número de registros de Hylocereus por estado y condición silvestre o cutivada.

\begin{tabular}{|c|c|c|c|c|c|c|c|c|c|c|}
\hline \multirow[t]{2}{*}{ Estado } & \multicolumn{2}{|c|}{ H. aff. escuintlensis } & \multicolumn{2}{|c|}{ H. ocamponis } & \multicolumn{2}{|c|}{ H. purpusii } & \multicolumn{2}{|c|}{ H. undatus } & \multicolumn{2}{|c|}{ Hylocereus sp. } \\
\hline & $\mathrm{S}$ & $\mathrm{C}$ & $\mathrm{S}$ & $\mathrm{C}$ & $\mathrm{S}$ & $\mathrm{C}$ & $\mathrm{S}$ & $\mathrm{C}$ & $\mathrm{S}$ & $\mathrm{C}$ \\
\hline Campeche & & & & & & & 3 & & & \\
\hline Chiapas & 4 & 8 & & & & & 24 & 21 & & \\
\hline Colima & & & 1 & & 1 & & & 1 & & \\
\hline Distrito Federal & & & & & & 1 & & & & \\
\hline Guanajuato & & & & & & & 4 & 2 & & \\
\hline Guerrero & & & 4 & 1 & 6 & & 2 & 1 & & \\
\hline Hidalgo & & & & & & & 7 & 2 & & \\
\hline Jalisco & & & 13 & 13 & 15 & 14 & 3 & 10 & & \\
\hline Edo. de México & & & & & & 1 & 3 & 1 & & \\
\hline Michoacán & & & 9 & 8 & 11 & 3 & 1 & & & \\
\hline Morelos & & & & & & & & 1 & & \\
\hline Nayarit & & & & & 38 & 10 & & 1 & & \\
\hline Oaxaca & & & & & 3 & 1 & 23 & 17 & 3 & 4 \\
\hline Puebla & & & & & & & 7 & 10 & & \\
\hline Querétaro & & & & & 1 & 1 & 9 & 6 & & \\
\hline Quintana Roo & & & & & & & & 1 & & \\
\hline San Luis Potosí & & & & & 1 & 1 & 7 & & & \\
\hline Sinaloa & & & & & 4 & 4 & & & & \\
\hline Tabasco & & & & & & & 12 & 1 & & \\
\hline Veracruz & & & & & & & 47 & 3 & & \\
\hline Yucatán & & & & & & & 3 & & & \\
\hline Total & 4 & 8 & 27 & 22 & 80 & 36 & 155 & 78 & 3 & 4 \\
\hline
\end{tabular}

$\mathrm{S}=$ Silvestre, $\mathrm{C}=$ Cultivada 

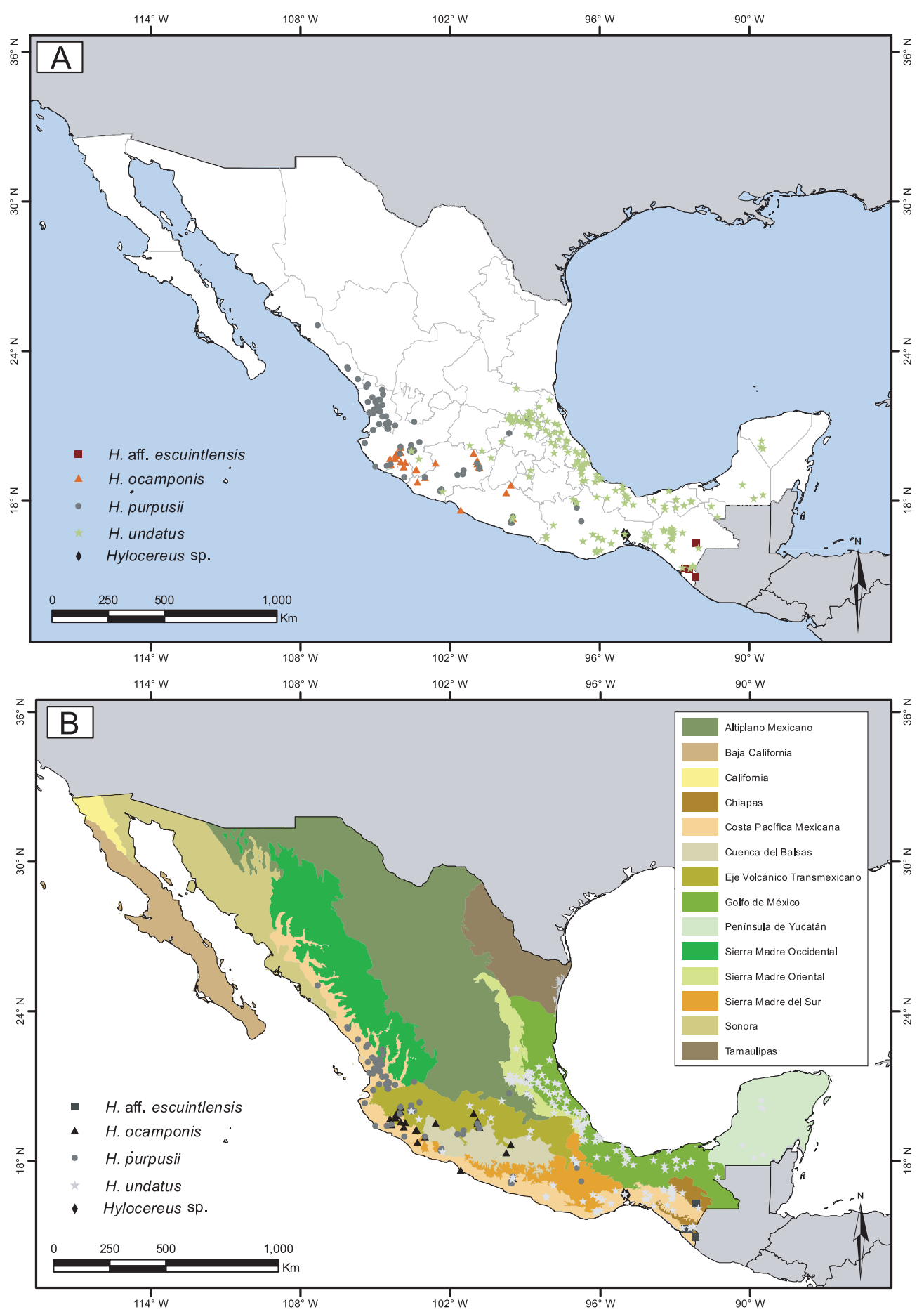

Figura 1. Distribución de Hylocereus en México. A) por entidad federativa, B) por Provincias biogeográficas.

Centro y Sureste del país (Figura 1A). Hylocereus undatus crece en 15 estados, $H$. purpusii en nueve, $H$. ocamponis en cuatro e $H$. aff. escuintlensis en uno (Tabla 1). Hylocereus crece en 11 de las 14 provincias biogeográficas propuestas para México (Figura 1B). Las cuatro especies se desarrollan a lo largo de la provincia Costa Pacífica Mexicana (166 registros). Hylocereus ocamponis, H. purpusii e H. undatus confluyen en el Eje Volcánico Transmexicano (90), Cuenca del Balsas (18) y Sierra Madre del Sur (18). En la Sierra Madre Occidental crece únicamente H. purpusii (3). En contraste, $H$. undatus se encuentra en nueve provincias (Figura 1B), creciendo con más abundancia en el Golfo de México (72), Sierra Madre Oriental (20) y Península de Yucatán (6). Por su parte Hylocereus aff. escuntlensis se distribuye en la 
Luz Adriana García-Rubio et al.

Tabla 2. Subcuencas hidrográficas con mayor número de especies y registros de Hylocereus en México.

\begin{tabular}{|c|c|c|c|c|c|c|}
\hline & Subcuenca hidrográfica & H. aff. escuintlensis & H. ocamponis & H. purpusii & H. undatus & Hylocereus sp. \\
\hline 1 & $\begin{array}{l}\text { Medio Balsas, Alto Balsas, } \\
\text { Tepalcatepec }\end{array}$ & & 9 & 7 & 1 & \\
\hline 2 & Costa Chica de Guerrero & & 1 & 6 & 4 & \\
\hline 3 & Bajo Lerma & & 1 & 6 & 2 & \\
\hline 4 & $\begin{array}{l}\text { Rio Bajo Santiago; Rio } \\
\text { Alto Santiago }\end{array}$ & & & 15 & 1 & \\
\hline 5 & Rio Papaloapan & & & 2 & 13 & \\
\hline 6 & Costa de Jalisco & & 1 & 4 & & \\
\hline 7 & $\begin{array}{l}\text { Bajo Lerma, Alto Lerma, } \\
\text { Medio Lerma, La Laja }\end{array}$ & & & 1 & 2 & \\
\hline 8 & Rio Verde & & & 1 & 2 & \\
\hline 9 & $\begin{array}{l}\text { Alta-Grijalva, Grijalva- } \\
\text { Usumacinta, Usumacinta, } \\
\text { Bajo Grijalva }\end{array}$ & 1 & & & 33 & \\
\hline 10 & Costa de Chiapas & 3 & & & 5 & \\
\hline 11 & Rio Armería & & 10 & 3 & & \\
\hline 12 & $\begin{array}{l}\text { B. Panuco, A. Panuco, R. Tula, } \\
\text { R. San Juan Qro, R. Tulancingo }\end{array}$ & & & 2 & 30 & \\
\hline 13 & Rio Coahuayana & & 3 & & 1 & \\
\hline 14 & Alto Lerma & & 1 & & & \\
\hline 15 & Costa Grande de Guerrero & & 1 & & & \\
\hline 16 & Rio Ameca & & & 13 & & \\
\hline 17 & Presidio-San Pedro & & & 6 & & \\
\hline 18 & San Pedro, Rosa Morada & & & 7 & & \\
\hline 19 & Rio Huicicila & & & 2 & & \\
\hline 20 & Rio Culiacán & & & 1 & & \\
\hline 21 & Resto de La Región & & & 5 & & 7 \\
\hline 22 & Norte de Veracruz & & & 19 & & \\
\hline 23 & Actopan La Antigua & & & & 16 & \\
\hline 24 & Coatzacoalcos & & & & 5 & \\
\hline 25 & Costa de Oaxaca & & & & 5 & \\
\hline 26 & Rio Tehuantepec & & & & 6 & \\
\hline 27 & Grijalva-Usumacinta & & & & 2 & \\
\hline 28 & Valle de México & & & & 2 & \\
\hline 29 & Yucatán Norte & & & & 3 & \\
\hline 30 & Yucatan Este & & & & 1 & \\
\hline
\end{tabular}

provincia de Chiapas (10). Hylocereus sp. se restringe a la Costa Pacífica Mexicana.

En cuanto a los tipos de vegetación donde crece Hylocereus se aprecian dos tendencias: H. ocamponis e H. purpusii predominan en bosques tropicales caducifolios (Btc) y bosque de coníferas y encinos (Bce) hacia el occidente del país. Hylocereus undatus se desarrolla hacia el centro y sureste del país en bosques tropicales perennifolio (Btp), BTC y BT subcaducifolio (BTsC). Hylocereus aff. escuintlensis crece en Btp y Btc, sólo se ubicó en Bce en Ocosingo, Chiapas (Figura 2A). Para Hylocereus sp. se registró su desarrollo en el Btc y en el bosque espinoso (Be).

Las especies de Hylocereus se desarrollan a una altitud de 0-2,496 m s.n.m., precipitación media anual de 400-4,000 mm y temperatura media anual de 12 a $28{ }^{\circ} \mathrm{C}$. Esto abarca los gradientes templado, semicálido, cálido y muy cálido (Figura 2B). Hylocereus ocamponis e H. purpusii coexisten en áreas con condiciones ecológicas similares, con temperaturas de 16 a $26^{\circ} \mathrm{C}$, desde el nivel del mar hasta los 2,160 m (H. ocamponis de 80 a 2,160 e H. purpusii de 2 a 2,121 m s.n.m.) y un gradiente de precipitación que va de 400 a 2,500 $\mathrm{mm}$. Por su parte, $H$. undatus exhibe el límite de los intervalos mínimo y máximo referidos en altitud y temperatura e intervalo de precipitación más amplio (600-4,000 mm). Esta especie prospera junto con H. ocamponis e H. purpusii en todos los gradientes isotermales. En contraste, $H$. aff. escuintlensis se desarrolla en tierras bajas (0-479 m s.n.m.), más cálidas $\left(18-28^{\circ} \mathrm{C}\right)$ con 800 a 4,000 mm de precipita- 

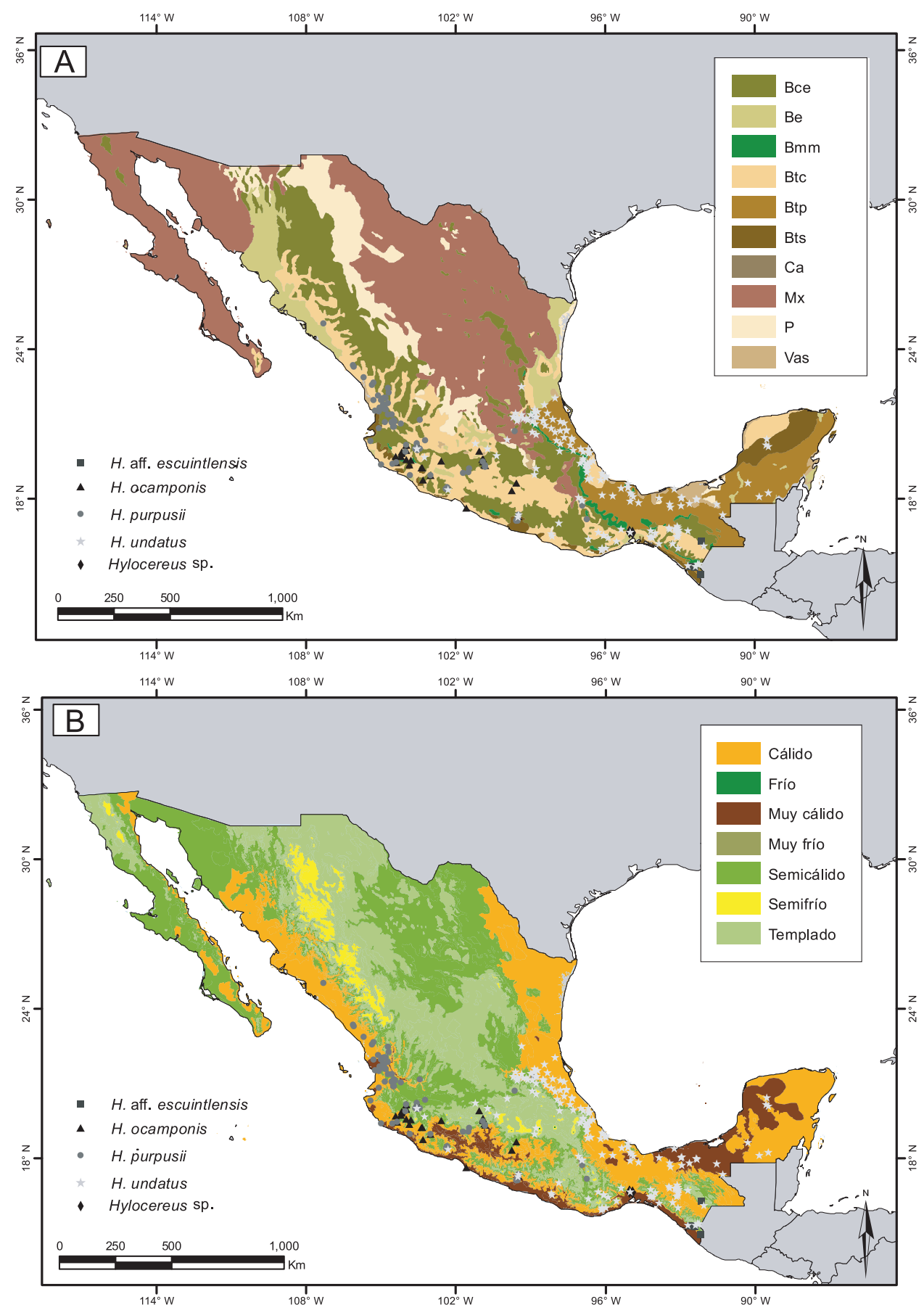

Figura 2. Distribución de Hylocereus en México. A) por tipos de vegetación y B) Isotermas medias anuales. Abreviaturas de tipos de vegetación: Bce. Bosque de coníferas y encinos, Be. Bosque espinoso, Bmm. Bosque mesófilo de montaña, Btc. Bosque tropical caducifolio, Btp. Bosque tropical perennifolio, Bts. Bosque tropical subcaducifolio, Mx. Matorral xerófilo, P. Pastizal, Vas. Vegetación Acuática y Subacuatica.

ción. Esta especie no se encuentra en las partes altas de las montañas con ambientes templados, si no que se desarrolla en las laderas bajas de las montañas con ambiente más cálido. Finalmente, Hylocereus sp. es también de bajas altitudes de 35 a $80 \mathrm{~m}$ e isotermas que van de los 20 a los $24{ }^{\circ} \mathrm{C} \mathrm{y}$ precipitación de 800 a los $1,500 \mathrm{~mm}$.

Hylocereus se ubica en 30 de las 70 subcuencas hidroló- gicas propuestas para México (Tabla 2). Hylocereus ocamponis, $H$. purpusii e $H$. undatus, concurren en tres: Medio Balsas-Alto Balsas-Tepalcatepec, Costa Chica de Guerrero y Bajo Lerma, con el mayor número de registros 17, 10 y 6 respectivamente. Catorce subcuencas cuentan sólo con un registro. Hylocereus ocamponis se ubicó de forma escasa y exclusiva en las subcuencas Alto Lerma y Costa Grande de 
Guerrero (4 registros) mientras que $H$. purpusii se presenta de forma única en 16 subcuencas e $H$. undatus en 22 . El número de registros para $H$. purpusii fue más alto en Rio Ameca (15) y para $H$. undatus en Alta Grijalva- Grijalva Usumacinta-Usumacinta-Bajo Grijalva (33). Por su parte $H$. aff. escuintlensis se ubica en las subcuencas Alta-Grijalva, Grijalva-Usumacinta, Usumacinta, Bajo Grijalva y Costa de Chiapas mientras que Hylocereus sp. abarca sólo la subcuenca Resto de La región.

\section{Discusión}

Se reconoce la presencia de cuatro taxa: $H$. ocamponis, $H$. purpusii, $H$. undatus e $H$. aff. escuintlensis. Las tres primeras han sido identificadas para el país, a partir de estudios taxonómicos (Bravo-Hollis, 1978; García-Aguilar et al., 2009; Bravo-Hollis y Arias, 2011). Hylocereus undatus presenta cierta plasticidad fenotípica, no obstante los atributos diagnósticos que facilitan su distinción son tallos de color verde, sin presencia de cera, areolas con espinas de 2 a 3 , de 2-4 $\mathrm{mm}$ de largo y $1 \mathrm{~mm}$ ancho en la base, flores con brácteas anchamente ovadas verde claro, tépalos externos ancho-lineares verde amarillentos y tépalos internos anchamente lanceolados, blancos a amarillentos (Arias et al., 1997). Por su parte, H. purpusii e H. ocamponis son dos taxa distintos. En las síntesis taxonómicas ofrecidas para este género Bauer (2003) y Hunt (2006) incluyen a $H$. purpusii como sinónimo de $H$. ocamponis, especie cuya descripción es más antigua; esta decisión la fundamentan en la similitud morfológica entre ambos taxa y el escaso material disponible. No obstante, hay diferencias en la anatomía caulinar (grosor de la cutícula e hipodermis, tamaño de los radios de la madera), morfológicas en areolas (diámetro, longitud y forma de espinas) y estructuras florales que sustentan su distinción (Bravo-Hollis, 1978; García-Aguilar et al., 2009; Corona-Oceguera, 2012). Además, la diferenciación entre $H$. ocamponis, $H$. purpusii e $H$. undatus fue ratificada con el análisis de secuencias de tres regiones de cloroplasto (matK, $r b c L, p s b A$ ) por García-Aguilar et al. (2013).

Por su parte, H. escuintlensis fue citada para el sureste sin especímenes de referencia que precisaran su ubicación (Cálix de Dios, 2004). Durante el trabajo de campo realizado para este estudio, colectamos plantas de Hylocereus en doce localidades de Chiapas (Apéndice 1) que morfológicamente coinciden con la descripción tipo de H. escuintlensis (Kimnach, 1984) en los siguientes rasgos: planta trepadora, de 3 a $4 \mathrm{~m}$ de longitud o mayor, tallos de tres ángulos con caras cóncavas, de 3.2 a $6 \mathrm{~cm}$ ancho (Figura 3). Presenta 1 a 2 (-3) espinas en forma de punzón con las bases engrosadas, de 1.1 a $1.5 \mathrm{~mm}$ de longitud, de color café claro. Sólo una de

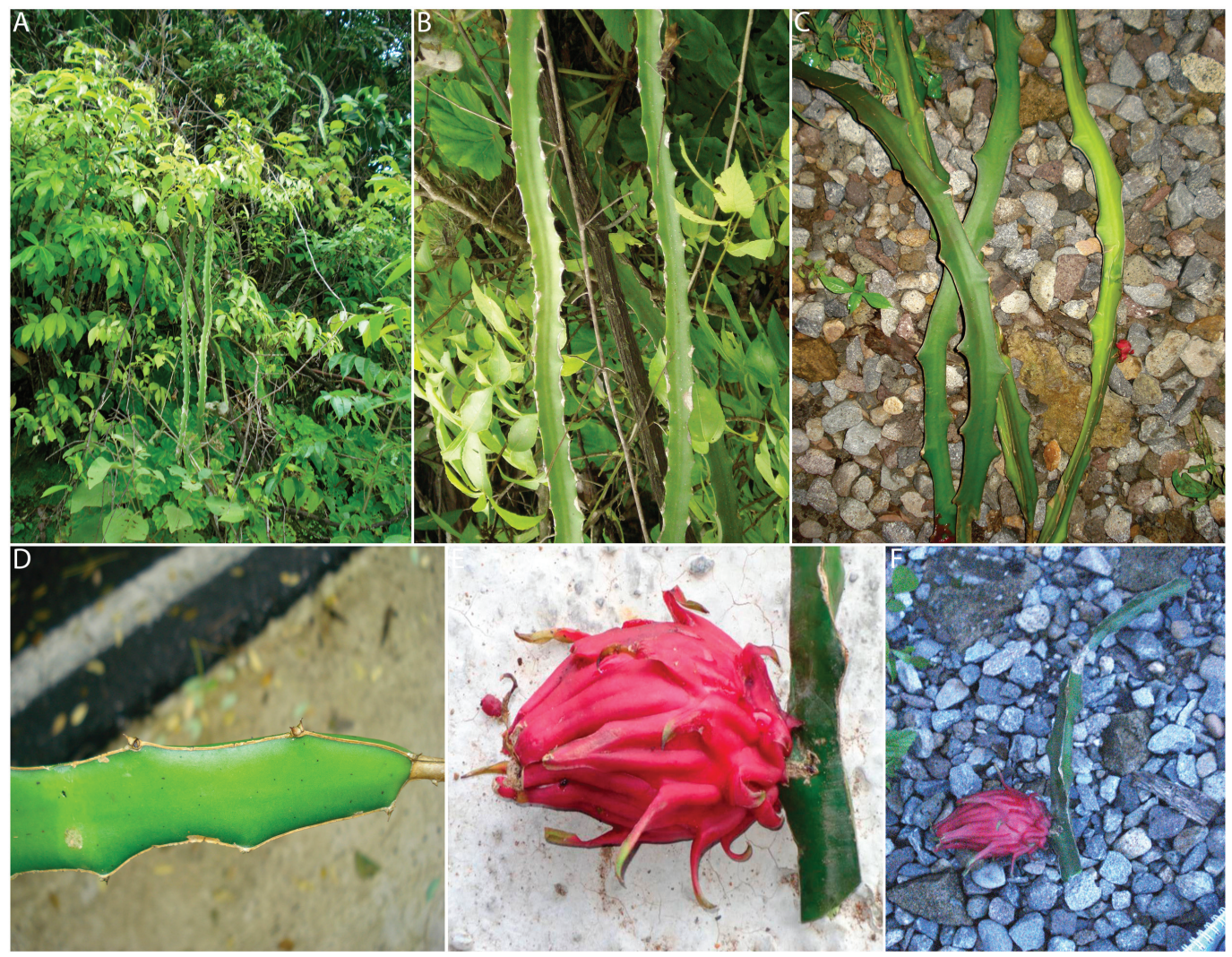

Figura 3. Hylocereus aff. escuintlensis en Chiapas. A) vista del hábitat y B) planta (tallos recolectados en San Bartolo, Ocosingo); C) tallos; cicatriz del fruto; D) espinas del tallo, (recolecta de Cintalapa, Escuintla); E) y F) fruto (recolectado en El Trapiche, Tuxtla Chico. 


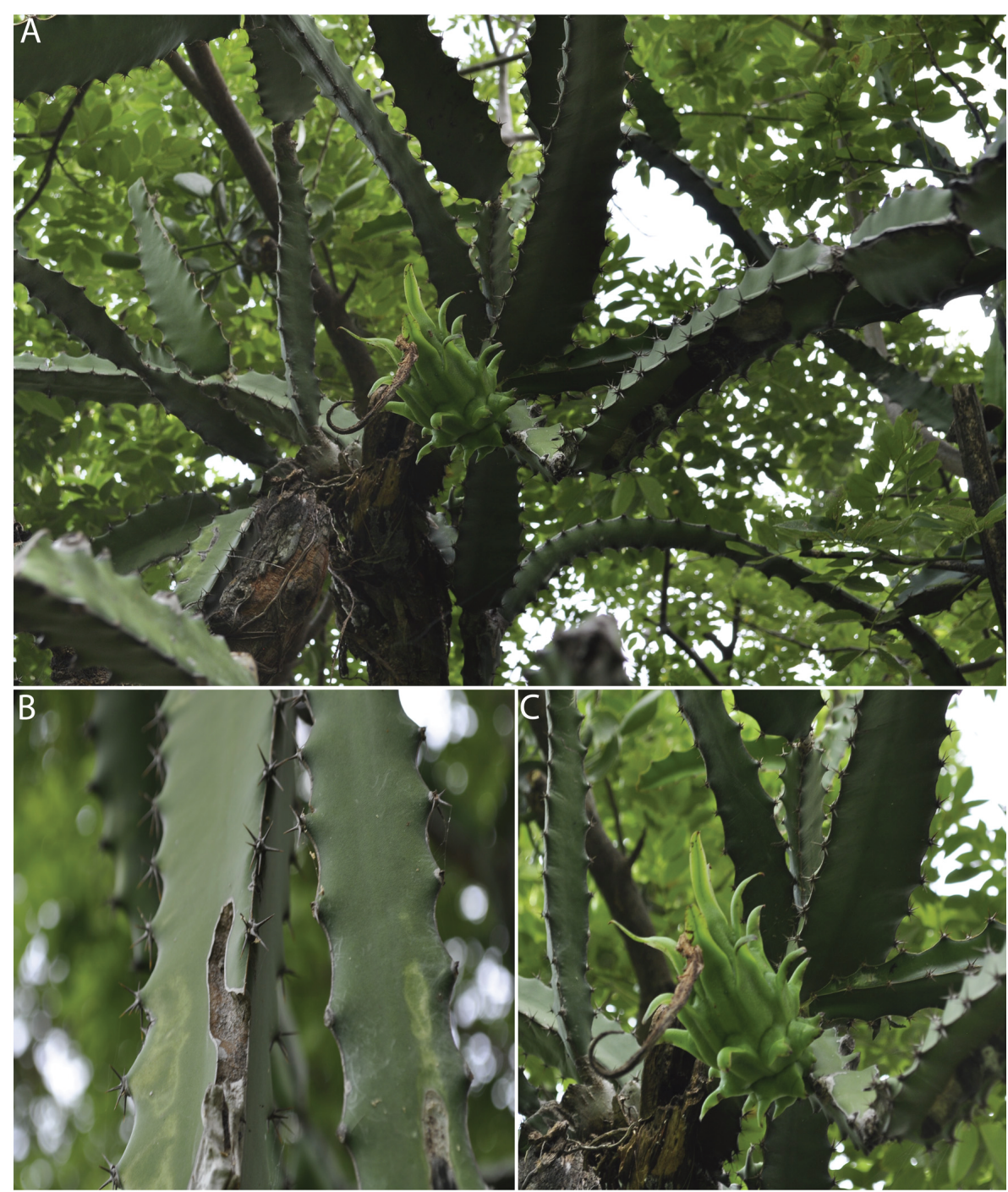

Figura 4. Hylocereus sp. silvestre en Oaxaca. A) vista del hábitat; B) tallo (areolas); C) fruto inmaduro (recolectado en Nisanda, Asunción Ixtaltepec).

las plantas colectadas presentaba un fruto de forma ovada, de $8.7 \mathrm{~cm}$ de longitud y $5.8 \mathrm{~cm}$ de ancho, con exocarpo de color rojo-purpura y brácteas en forma de podarios aplanados, fuertemente recurvadas en la mitad inferior del fruto, las inferiores son ovadas y las superiores son lanceoladas de $3.2 \mathrm{~cm}$ de largo y $3.4 \mathrm{~cm}$ de ancho (Figura 3). Estos atributos coinciden con lo señalado en la descripción original (Kimnach, 1984) y ejemplares de Guatemala (Véliz Pérez, 2011), así como con los rasgos de los isotipos de H. escuintlensis depositados en las colecciones de los herbarios K, US, F y en la colección UNIBIO del Instituto de Biología de la Universidad Nacional Autónoma de México [fotografías disponibles y revisadas de los especímenes Birdsey M. 313B, Sheet 1329 (K); Birdsey M. 313, Sheet 2717459, barcode 00115679 (US); Birdsey M. $313 b$ catalog number 1588908, barcode V0052880F (F); Birdsey M. 313 número de catálogo PVT1283225 (UNIBIO)]. Sin embargo, debi- do a que la recolecta de estos materiales se realizó después de la temporada de floración y fructificación (septiembre a noviembre) no se tienen datos de la morfología floral, del color del endocarpo y la variación que puede exhibir los frutos (dimensión, morfología), por lo que estos materiales se determinaron como afines a H. escuintlensis. Los especímenes colectados se encuentran bajo cultivo para observar los atributos reproductivos y preparar ejemplares de herbario. La existencia de $H$. escuintlensis en el sureste de México es esperada, ya que el material tipo fue descrito del área vecina de Escuintla, Guatemala (Kimnach, 1984), a partir de pocos ejemplares que provienen de áreas muy cercanas ubicadas a mayor altitud que los ejemplares colectados en Chiapas.

Las plantas de Hylocereus recolectados en siete localidades de Oaxaca catalogadas como Hylocereus sp. presentan atributos morfológicos distintos como son bordes del tallo convexos, espinas numerosas, el fruto inmaduro es de forma 
ovoide-globoso y presenta brácteas triangulares, las inferiores son cortas, tan largas como anchas, con la base foliar dilatada, algo retrorsas y hacia el ápice del fruto las brácteas son alargadas y de mayor longitud (Figura 4). El endocarpo en la madurez es blanco. No se observaron flores. Actualmente se encuentran en cultivo para observar y registrar sus características y determinar a qué especie pertenecen.

La presencia de Hylocereus minutiflorus en México no se corroboró. El espécimen colectado por Martínez Camilo 786 determinado como H. minutiflorus y citado para Chiapas (Guzmán et al., 2003) fue cotejado en el herbario HEM de la Universidad de Ciencias y Artes de este estado. Se trata de un ejemplar único, que consta sólo de material vegetativo (tallos) y carece de flores y frutos, por lo que no fue posible ratificar la determinación. Tampoco se localizó en el sitio de colecta referido (ejido la Alianza, Reserva la Sepúltura, Mpio. Arriaga). No obstante, la presencia de H. minutiflorus al igual que la de $H$. escuintlensis es esperada ya que su límite norte de distribución es Guatemala (Anderson, 2001). Finalmente, durante esta investigación, en los herbarios revisados y en las exploraciones de campo, no se encontraron ejemplares que correspondan a $H$. napoleonis (actualmente como $H$. trigonus), H. calcaratus, H. guatemalensis, H. stenopterus, H. trigonus e $H$. triangularis, especies de afinidad centroamericana (Britton y Rose, 1920, 1963; Anderson, 2001; Hunt, 2006). Para reconocer o descartar su presencia (incluyendo a $\mathrm{H}$. minutiflorus) se requiere mayor exploración en áreas del sureste de México donde existan condiciones ecológicas potenciales para su desarrollo; acción que deberá complementarse con la revisión de ejemplares de Hylocereus en las colecciones internacionales (incluyendo los tipos).

Hylocereus sigue dos grandes patrones de distribución geográfica en el territorio mexicano. Hacia el occidente se desarrollan principalmente $H$. ocamponis e $H$. purpusii, mientras que en el sureste se encuentran $H$. undatus, $H$. aff. escuintlensis e Hylocereus sp. (Figura 1A). Por su parte, $H$. ocamponis, $H$. purpusii e $H$. undatus llegan a coexistir. Sin embargo, $H$. ocamponis e $H$. purpusii no crecen en los mismos sitios que $H$. aff. escuintlensis e Hylocereus sp. debido a diferencias en los requerimientos ecológicos. Para $H$. undatus se registra su distribución en una extensa área que abarca el centro y sureste de la República. Se recolectaron especímenes silvestres de esta especie en Campeche, Tabasco y Yucatán, áreas donde se indicaba su presencia (Cálix de Dios, 2004; Castillo-Martínez, 2006) y se observaron en la huasteca potosina. Hylocereus aff. escuintlensis se registró únicamente en Chiapas. Hylocereus ocamponis se limita a los estados de Colima, Guerrero, Jalisco y Michoacán, no se confirma su presencia como silvestre en Morelos y el Estado de México indicada por Cálix de Dios (2004). Hylocereus purpusii tiene una distribución más amplia, el límite norte es Sinaloa extendiéndose al sur a través de Nayarit, Jalisco, Colima, Michoacán, Guerrero y Oaxaca. No se encontró como silvestre en el Estado de México y Durango (a partir de los ejemplares de herbario revisados) como refiere Cálix de Dios (2004). Hylocereus ocamponis e H. purpusii son nativas de México, aunque $H$. ocamponis es referida por Britton y Rose (1920) como silvestre para Colombia. A pesar de que el origen de $H$. undatus es impreciso (Backeberg, 1976; Bravo-Hollis, 1978; Anderson, 2001; Hunt, 2006) su frecuencia natural en el golfo de México y distribución hacia el sureste sugiere que México es un área de diversificación de este taxon, donde se manifiesta amplia variabilidad morfológica y genética (Legaria et al., 2005). Los patrones de distribución encontrados utilizando un SIG confirman los señalados por García-Aguilar (2007) y los inferidos por Cálix de Dios (2004) con otras herramientas.

A grandes rasgos se observan dos tendencias en la distribución de Hylocereus por provincia biogeográfica en México: (1) H. purpusii, H. ocamponis e H. undatus crecen en las provincias Costa Pacífica Mexicana, Eje Volcánico Transmexicano, Cuenca del Balsas y Sierra Madre Occidental, en el Centro y Occidente del país; (2) $H$. aff. escuintlensis, $H$. undatus e Hylocereus sp. se desarrollan en las provincias Sierra Madre Oriental, Golfo de México, Costa Pacífica Mexicana, Chiapas y Península de Yucatán hacia el Sureste (Figura 1B). La Costa Pacífica Mexicana tiene el mayor número de especies (5) y ha permitido que un género con afinidad Neotropical como Hylocereus llegue hasta Sinaloa. Por otro lado, H. ocamponis, H. purpusii e H. undatus convergen en las provincias Sierra Madre del Sur y en el Eje Volcánico Transmexicano, las cuales han actuado como corredores en la distribución de estas especies. Sin embargo, esta última provincia es a su vez una barrera física y climática que limita la dispersión de Hylocereus hacia el norte por el centro del país, con excepción de $H$. undatus y H. ocamponis que se encuentran en el Altiplano Mexicano. Hylocereus undatus es la especie más ampliamente distribuida siguiendo dos vías: (1) Costa Pacífica y (2) Golfo de México en la cual está más representada, ya que en esta área ha encontrado las condiciones ecológicas propicias para su desarrollo. Ésta distribución divergente es el patrón natural de la especie, dado que la modelación se hizo considerando sólo materiales silvestres. Por su parte $H$. aff. escuintlensis se restringe a la porción sur de la Costa Pacífica Mexicana y partes bajas de Chiapas $(<500 \mathrm{~m})$ en donde hay áreas cálidas que cubren sus demandas ecológicas. Finalmente, Hylocereus sp. crece en la Costa Pacífica Mexicana, dentro de esta provincia tiene hábitats muy específicos. En resumen las provincias biogeográficas han funcionado como corredores y en algunos casos como barreras en la dispersión de Hylocereus en México.

Hylocereus undatus tiene una amplia capacidad de adaptación ecológica y se encontró en distintos ambientes. Por su parte, $H$. ocamponis e $H$. purpusii tienen requerimientos ecológicos similares, no obstante $H$. purpusii tolera mayor precipitación (400-2,500 mm versus 400-2,000 mm). Hylocereus aff. escuintlensis mostró una tendencia a prosperar en 
ambientes del trópico húmedo, con niveles de precipitación altos $(800-4,000 \mathrm{~mm})$, temperaturas cálidas $\left(18-28^{\circ} \mathrm{C}\right)$ y en áreas de baja altitud (no mayor a los $500 \mathrm{~m}$ ). Hylocereus $\mathrm{sp}$. presenta los rangos más estrechos de altitud (no mayor a los $80 \mathrm{~m}$ ), temperatura (de los 20 a los $24^{\circ} \mathrm{C}$ ) y precipitación media anual de 800 a los $1,500 \mathrm{~mm}$; empero, mayor exploración en el sureste del país ayudará a precisar la amplitud ecológica que tolera esta especie. Los valores mínimos y máximos de las variables de altitud, precipitación media anual y temperatura media anual registradas en los hábitats donde se desarrolla Hylocereus (0-2,496 m, 400-4,000 $\mathrm{mm}$ y $\left.12-28^{\circ} \mathrm{C}\right)$, concuerdan con los señalados por Cálix de Dios (2004) (2-2750 m, 400-4,000 mm y $\left.13-28{ }^{\circ} \mathrm{C}\right)$. Se confirma que Hylocereus crece en Mesoamérica en una variedad de paisajes como propusieron Ortiz-Hernández y Carrillo-Salazar (2012).

Las subcuencas hidrológicas Medio Balsas-Alto BalsasTepalcatepec, Costa Chica de Guerrero y Bajo Lerma albergan la mayor riqueza de Hylocereus. No hay una correlación entre la extensión que cubren y el número de registros en cada una. Las condiciones climáticas de éstas $\left(16-22{ }^{\circ} \mathrm{C}\right.$, 600-1,019 mm, clima cálido subhúmedo y semicálido subhúmedo) (Toledo, 2003; Patiño-Gómez et al., 2012; CONAGUA, 2005) determinan la confluencia de $H$. ocamponis, $H$. purpusii e $H$. undatus. Los tipos de vegetación que cubren estas áreas son Btc, Bts, Bce y en menos proporción BE en La Costa Chica de Guerrero (CONAPO, 2000; Toledo, 2003; Patiño-Gómez et al., 2012). No obstante, los límites de los microhábitats en que pueden habitar estas especies pueden ser más amplios, lo que se evidencía al registrarse su presencia en 30 de las 70 subcuencas que hay en México. Futuras exploraciones de campo ayudarán a identificar las subcuencas y condiciones climáticas donde puede desarrollarse $H$. aff. escuitlensis e Hylocereus sp. Asimismo, el uso de herramientas como el modelado de nicho ecológico sería de gran utilidad.

Finalmente, la revisión de los ejemplares de Hylocereus en los herbarios y colecciones o jardines botánicos nacionales consultados muestra que la representatividad de las especies es aún escasa y a menudo los especímenes son incompletos lo que puede llevar a identificaciones erróneas. Se requiere continuar con la exploración y colecta en ciertas áreas del país, al norte desde Sinaloa, los estados de México y Querétaro en el centro, Colima, Michoacán en el occidente y Oaxaca y Chiapas en el sureste del país. Esto contribuirá a tener una mejor representación de la riqueza de especies de Hylocereus. De igual forma las nuevas colectas podrán contener datos de latitud y longitud; la conjunción de esta información, riqueza y distribución, podrá ser utilizada en futuros estudios biogeográficos y biosistemáticos, que ayuden a entender la historia natural de Hylocereus. Asimismo, el conocimiento básico de la riqueza, patrones de distribución y factores ecológicos que determinan la misma son un referente importante para conducir estudios futuros sobre Hyloce- reus. Estos podrían dirigirse a registrar la importancia cultural y utilización de las cuatro especies que crecen en México. Abordando además estudios de caracterización morfológica, genética y documentar el proceso de domesticación de las especies que son manejadas in situ y cultivadas.

\section{Agradecimientos}

Agradecemos a los revisores sus atinados comentarios y sugerencias para la mejora del manuscrito. Agradecemos a los curadores de los herbarios consultados las facilidades brindadas. A Walter Zabala por el apoyo en las colectas de campo. El financiamiento fue otorgado a OVP por la SAGARPA-SINAREFI y a LAGR por el CONACYT como beca de posgrado.

\section{Literatura citada}

Anderson E.F. 2001. The Cactus Family. Timber Press, Portland. Arias M.S., Gama-López S., Guzmán-Cruz L.U. y Vázquez-Benítez B. 1997. Cactaceae Juss. Flora del Valle de Tehuacán-Cuicatlán, Fascículo 95. Instituto de Biología, Universidad Nacional Autónoma de México. México D.F.

Awang Y., Ghani M.A.A., Sijam K. y Mohamad R.B. 2011. Effect of calcium chloride on anthracnose disease and postharvest quality of red-flesh dragon fruit (Hylocereus polyrhizus). African Journal of Microbiological Research 5:5250-5259.

Backeberg C. 1976. Cactus lexicon. Enumeratio diagnostica cactacearum. Blandford Press, Dorset.

Barthlott W. y Hunt D.R. 1993. Cactaceae. En: Kubitzki K., Rohwer J.G., y Bittrich V. Eds. The families and genera of vascular plants, Vol. II, Flowering Plants. Dicotyledons: Magnoliid, Hamamelid and Caryophyllid families, pp:161-197, SpringerVerlag, Heidelberg.

Bauer R. 2003. A synopsis of the tribe Hylocereeae F. Buxb. (Cactaceae). Systematics Initiatives 17:1-63.

Ben-Asher J., Nobel P.S., Yossov E. y Mizrahi Y. 2006. Net CO2 uptake rates for Hylocereus undatus and Selenicereus megalanthus under field conditions: Drought influence and a novel method for analyzing temperature dependence. Photosynthetica 44:181-186.

Bravo-Hollis H. 1978. Las Cactáceas de México, Vol. 1. Universidad Nacional Autónoma de México. México D.F.

Bravo-Hollis H. y Arias S. 2011. Cactaceae. En: Flora Mesoamericana, Vol. 2, parte 1. Universidad Nacional Autónoma de México, México D.F. Disponible en: <http://www.tropicos.org/docs/ meso/cactaceae.pdf $>$ (consultado 7 mayo 2014).

Britton N.L. y Rose J.N. 1920. The Cactaceae: descriptions and illustration of plants of the cactus family., Vol. II. The Carnegie Institution, Washington D.C.

Britton N.L. y Rose J.N. 1963. The Cactaceae: descriptions and illustration of plants of the cactus family, Vol. I y II. Dover Publications Inc., Nueva York.

Cálix de Dios H. 2004. Distribución geográfica de las pitahayas (Hylocereus) en la república mexicana. Cactáceas y Suculentas Mexicanas 49:4-23.

Castillo-Martínez R. 2002. Caracterización morfológica, reproductora y fisiológica de genotipos de Hylocereus undatus (Cacta- 
ceae) de la península de Yucatán. Tesis de doctoral, Facultad de Ciencias, Universidad Nacional Autónoma de México, México D.F. 93 pp.

Castillo-Martínez R. 2006. Aprovechamiento de la pitahaya: bondades y problemáticas. Caos Conciencia 1:13-18.

Castillo-Martínez R., Livera M.M., Brechú F.A.E. y Márquez-Guzmán J. 2003. Compatibilidad sexual entre dos tipos de Hylocereus (Cactaceae). Revista Biología Tropical 51:699-705.

Cisneros A. y Tel-Zur N. 2010. Embryo rescue and plant regeneration follow interspecific crosses in the genus Hylocereus (Cactaceae). Euphytica 174:73-82.

Cisneros A. y Tel-Zur N. 2013. Genomic analysis in three Hylocereus species and their progeny: evidence for introgressive hybridization and gene flow. Euphytica 194:109-124.

CONAGUA. 2005. Formulación del programa hídrico por organismo de cuenca, visión 2030, de la región hidrológico-administrativa VIII, Lerma-Santiago-Pacífico. Resumen ejecutivo. Comisión Nacional del Agua. Disponible en: <http://www.conagua.gob.mx/OCLSP07/NotaP/FINALaaResumenEjecutivo. pdf $>$ (consultado 22 Enero 2014).

CONAPO. 2000. Población, medio ambiente y desarrollo sustentable: dos estudios de caso: regiones Mariposa Monarca y Costa Chica de Guerrero. Secretaria de Medio Ambiente, Recursos Naturales y Pesca, Consejo Nacional de Población y Facultad Latinoamericana de Ciencias Sociales, México D.F.

Corona-Oceguera C.A. 2012. Variación morfológica de Hylocereus (Cactaceae) en Jalisco. Tesis de Licenciatura, Universidad de Guadalajara, Zapopan. 76 pp.

Cronquist A. 1982. Map of the floristic provinces of North America. Britonnia 34:144-145.

ESRI [Enviromental Systems Research Institute]. 1992-2002. Arc View-Gis 3.3. Enviromental Systems Research Institute, Redlands.

García-Aguilar M.A. 2007. Anatomía y morfología de las especies silvestres del género Hylocereus (Berger) Britton y Rose (Cactaceae) en México. Tesis de Maestría, Colegio de Posgraduados, Montecillo, Texcoco. 58pp.

García-Aguilar M.A, Terrazas T. y Arias S. 2009. Anatomía caulinar de tres especies del género Hylocereus (Berger) Britton \& Rose (Cactaceae) en México. Revista Fitotecnia Mexicana 32:201-208.

García-Aguilar M.A., Terrazas T., Segura-León O., Arias S., Vibrans H. y López-Mata L. 2013. Caracterización molecular de tres especies de Hylocereus (Cactaceae) presentes en México. Revista Fitotecnia Mexicana 36:13-22.

García-E y CONABIO. 1998. Isotermas medias anuales, escala 1:1000000. Comisión Nacional para el Conocimiento y Uso de la Biodiversidad, México D.F.

Grimaldo-Juárez O., Terrazas T., García-Velásquez A., Cruz-Villangas M. y Ponce-Medina J.F. 2007. Morphometric analysis of 21 Pitahaya (Hylocereus undatus) genotypes. Journal of the Professional Association for Cactus Development 9:99-117.

Guzmán U., Arias S. y Dávila P. 2003. Catálogo de Cactáceas Mexicanas. Universidad Nacional Autónoma de México y Comisión Nacional para el Uso y Conocimiento de la Biodiversidad, México D.F.

Hart G. 2005. From prickly pear to dragon fruit: the changing face of cactus-fruit growing. Cactus and Succulent Journal 77:293-299.

Hoa T.T., Clark C.J., Waddell B.C. y Woolf A.B. 2006. Postharvest quality of Dragon fruit (Hylocereus undatus) following disin- festing hot air treatments. Postharvest Biology and Technology 41:62-69.

Hunt D.R. (Comp.). 1999. CITES Cactaceae checklist, 2nd ed. Royal Botanic Gardens, Kew.

Hunt D.R. 2006. The new Cactus Lexicon. DH books, Milborne Port.

INEGI-INE-CONAGUA. 2007. Mapa de cuencas hidrográficas de México (escala 1: 250000). Producto cartográfico derivado de la obra primigenia INE (2003) "Cuencas Hidrográficas de México, escala 1:250,000.”, elaborada por Priego A.G., Isunza E., Luna N. y Pérez J.LINTA. 2014. Guía Tecnológica 6: Cultivo de la Pitahaya. Instituto Nicaranguense de Tecnología Agropecuaria. Disponible en: http://www.inta.gob.ni/biblioteca/images/pdf/guias/GUIA\%20PITAHAYA\%202014.pdf

Jiang Y.-L., Liao Y.-Y., Lin T.-S., Lee Ch.-L., Yen. Ch.-R. y Yang W.-J. 2012. The photoperiod-regulated bud formation of red pitaya (Hylocereus sp.). HortScience 47:1063-1067.

Khaimov A. y Mizrahi Y. 2006. Effects of day-length, radiation, flower thinning and growth regulators on flowering of the vine cacti Hylocereus undatus and Selenicereus megalanthus. Journal of Horticultural Science \& Biotechnology 81:465-470.

Kimnach M.W. 1984. Hylocereus escuintlensis a new species from Guatemala. Cactus and Succulent Journal 56:177-179.

Le Bellec F., Vaillant F. e Imbert E. 2006. Pitahaya (Hylocereus spp.): a new fruit crop, a market with a future. Fruits 61:237-25.

Legaria-Solano J.P.., Alvarado-Cano M.E. y Gáspar-Hernández R. 2005. Diversidad genética en pitahaya Hylocereus undatus (Haworth) Britton y Rose. Revista Fitotecnia Mexicana 28:179-185.

Lichtenzveig J., Abbo S., Nerd A., Tel-Zur N. y Mizrahi Y. 2000. Cytology and mating system in the climbing cacti Hylocereus and Selenicereus. American Journal of Botany 87:10581065.

Maldonado-Poot L. 2000. Caracterización y clasificación fenotípica de pitahaya blanca Hylocereus undatus (Haworth) Britton y Rose del estado de Yucatán. Tesis Licenciatura, Centro Regional Universitario Península de Yucatán, Universidad Autónoma de Chapingo, Mérida. 119 pp.

Masyahit M., Sijam K., Awang Y. y Satar M.G. 2009. The first report of the occurrence of anthracnose disease caused by Colletotrichum gloeosporioides (Penz.) Penz. \& Sacc. on dragon fruit (Hylocereus spp.) in peninsular Malaysia. American Journal of Applied Sciences 6:902-912.

Miranda F. y Hernández X.E. 1963. Los tipos de vegetación de México y su clasificación. Boletín de la Sociedad Botánica de México 28:29-179.

Mizrahi Y. 2014. Vine-cacti pitayas - the new crops of the world. Revista Brasileira de Fruticultura 36:124-138.

Morrone J.J. 2005. Hacia una síntesis biogeográfica de México. Revista Mexicana de Biodiversidad 76:207-252.

Nerd A., Tel-Zur N. y Mizrahi Y. 2002. Fruits of vine and columnar cacti. En: Nobel P.S. Ed., Cacti: Biology and Uses. pp 185-197, University of California Press, Berkeley and Los Angeles.

Nobel P.S. y De la Barrera E. 2002. High temperatures and Net $\mathrm{CO}_{2}$ uptake, growth, and stem damage for the hemiepiphytic cactus Hylocereus undatus. Biotropica 34:225-231.

Nyffeler R. 2002. Phylogenetic relationships in the cactus family (Cactaceae) based on evidence from trnK/matK and trnL-trnF sequences. American Journal of Botany 89:312-326.

Ortiz-Hernández Y.D., Livera-Muñoz M. y Carrillo-Salazar A. 
1996. Asimilación de $\mathrm{CO}_{2}$ en tallos jóvenes de pitahaya (Hylocereus undatus). Revista Fitotecnia Mexicana 19:31-41.

Ortiz-Hernández Y.D., Livera-Muñoz M. y Carrillo-Salazar J.A. 1999. Asimilación de $\mathrm{CO}_{2}$ por la pitahaya (Hylocereus undatus) en condiciones de campo. Agrociencia 33:165-169.

Ortiz-Hernández Y.D. y Carillo-Salazar J.A. 2012. Pitahaya (Hylocereus spp.): a short review. Comunicata Scientiae 3:220-237.

Ortiz-Hernández Y.D., Livera-Muñoz M., Carrillo-Salazar J. A., Valencia-Botín A. y Castillo-Martínez R. 2012. Agronomical, physiological, and cultural contributions of pitahaya (Hylocereus spp.) in Mexico. Israel Journal of Plant Sciences 60:359-370.

Patiño-Gómez C., Reza-García I., Ruíz-Morelos B.-H., ZazuetaAcosta I., Gómez-Martínez J.F., Wagner-Gómez A., Rivera-Benítez J. y Balancán-Soberanis A. 2012. Portafolio de medidas de adaptación al cambio climático en el escurrimiento superficial de las regiones hidrológico administrativas de México. Proyecto de colaboración entre el Instituto Mexicano de Tecnología del Agua y el Instituto Nacional de Ecología. Disponible en: <http://www.inecc.gob.mx/descargas/cclimatico/2012_med_ adap_cc_reg_hidro.pdf $>$ (consultado 5 junio 2014).

Ramírez-Mireles F.J. 1999. Caracterización y compatibilidad en pitahaya Hylocereus sp. Tesis de Maestría en Horticultura, Universidad Autónoma Chapingo, Chapingo, Estado de México. 108 pp.

Raveh E., Nerd A. y Mizrahi Y. 1998. Responses of two hemiepiphytic fruit crop cacti to different degrees of shade. Scientia Horticulturae 73:151-164.

Rodríguez-Canto A. 2000. Pitahayas: Estado Mundial de su Cultivo y Comercialización. Fundación Yucatán Produce, A. C. Universidad Autónoma Chapingo, Texcoco.

Rzedowski J. 1978. Vegetación de México. Limusa, México D.F.

Rzedowski J. 1990. Vegetación potencial. IV.8.2. Atlas Nacional de México. Vol II. Escala 1: 4000 000. Instituto de Geografía, Universidad Nacional Autónoma de México, México D.F.

Serna-Cock L., Torres-Valenzuela L.S. y Ayala-Aponte A.A. 2011. Aplicación de 1-metilciclopropeno, una alternativa a la estacionalidad de la pitahaya amarilla. Alimentos Hoy 23:78-97.

Sotelo-Ruiz E.D., Ortiz-Trejo C. y Rizo-Arellano M.I. 2005. Áreas potenciales para el cultivo de Pitahaya (Hylocereus undatus (haw.) Britt. \& Rose) en el sur del estado de México. Revista Ciencia Forestal en México 30:87-97.

Tel-Zur N., Abbo S., Bar-Zvi D. y Mizrahi Y. 2004. Genetic relationships among Hylocereus and Selenicereus vine cacti (Cactaceae): evidence for hybridization and cytological studies. Annals of Botany 94:527-534.

Tel-Zur N., Mizrahi Y., Cisneros A., Mouyal J., Schneider B. y Doyle J.J. 2011. Phenotypic and genomic characterization of a vine cactus collection (Cactaceae). Genetic Resources and Crop Evolution 58:1075-1085.

Terrazas T. y Arias S. 2002. Comparative stem anatomy in the subfamily Cactoideae. The Botanical Review 68:444-473.

Toledo A. 2003. Ríos, costas, mares. Hacia un análisis integrado de las regiones hidrológicas de México. Secretaria de Medio Ambiente y Recursos Naturales e Instituto Nacional de Ecología, México D.F.

Valencia-Botín A.J., Sandoval-Islas J.S., Cárdenas-Soriano E., Michailides T.J. y Rendón-Sánchez G. 2003. Botryosphaeria dothidea causing stems spots on Hylocereus undatus in Mexico. Plant Pathology 52:803.

Valencia-Botín A.J., Sandoval-Islas J.S., Cárdenas-Soriano E., Michailides T.J. y Rendón-Sánchez G. 2004. A new stem spot disease of pitahaya (Hylocereus undatus H.) caused by Fusicoccum-like anamorph of Botryosphaeria dothidea in Mexico. Revista Mexicana de Fitopatología 22:40-42.

Valiente-Banuet A., Santos-Gally R., Arizmendi M.C. y Casas A. 2007. Pollination biology of the hemiepiphytic cactus Hylocereus undatus in the Tehuacán Valley, Mexico. Journal of Arid Environments 68:1-8.

Véliz Pérez M.E. 2011. Hylocereus minutiflorus Britton \& Rose (Cactaceae) una especie endémica de Mesoamérica. CactusAventures International 92:1-4.

Véliz Pérez M.E y Arias S. 2013. Hylocereus escuintlensis. The IUCN Red List of Threatened Species 2013. Disponible en: http:// www.iucnredlist.org/details/151976/0 (consultada 7 Mayo 2014).

Vidal-Zepeda R. 1990. Precipitación media anual, escala 1:4000000. En: Precipitación. Tomo II, Sección IV, 4.6. Atlas Nacional de México (1990-1992). Instituto de Geografía, Universidad Nacional Autónoma de México, México D.F.

Recibido: 17 de octubre de 2014

Aceptado: 28 de octubre de 2014 
Luz Adriana García-Rubio et aL.

Apéndice 1. Lista de especímenes de Hylocereus examinados. Las especies se presentan en orden alfabético. Los ejemplares examinados se organizan y presentan por condición de origen: silvestres, cultivados y desconocida. Se cita el estado, municipio, localidad, nombre del (los) colector (es) y número de colecta (en cursivas). Entre paréntesis se anotan las siglas del herbario en que se encuentra depositado el ejemplar de referencia. Nota: El símbolo de asterisco junto al nombre del herbario hace referencia a que la planta forma parte de la colección viva del instituto de Botánica de la Universidad de Guadalajara (IBUG*).

\section{Hylocereus ocamponis (Salm-Dyck) Britton \& Rose}

SILVESTRES. COLIMA, COMALA, Terrenos de la comunidad indígena Zacualpan, cerca de Campo Cuatro, R. Cuevas y J. Moreno 5846 (ZEA); GUERRERO, CHILPANCINGO DE LOS BRAVO, Rincón de la Vía, Kruse s.n. (MEXU); IXCATEOPAN, Carretera Iguala a Teloloapan, cerca de la desviación a los Sabinos, S. Gama et al. 104 (MEXU); PILCAYA, Grutas de Cacahuamilpa, J. Vázquez 1966, (MEXU); ZIHUATANEJO, Carretera Ixtápa Lázaro Cardenas, L. Scheinvar 4555 (MEXU); JALISCO, ATOYAC, Isla Grande, García-R. y Meza-C.1631 (IBUG*); AUTLÁN, Aserradero Tecopatlán, 19 km al S de El Chante, Rzedowski y McVaugh 1302 (ENCB); CASIMIRO CASTILLO, 1 Km al SO de Puerto Los Mazos, Carrillo-Reyes 6977 (IBUG*), CUAUTITLÁN DE GARCÍA BARRAGÁN, Brecha Cuautitlán-Ayotitlán, Ramírez-D. et al. 874 (IBUG); EJUTLA, San Lorenzo, Corona-O. y Vargas-P. 17 (IBUG*); Potrero de La Peña, 2.5 km al SO de Ejutla, Carrillo-Reyes y S. Monro y 6833 (IBUG*); EL LIMÓN, F.J. Santana y Arreola-N. 1449 (IBUG, ENCB); PIHUAMO, 3 Km al Este de Pihuamo, camino al rancho el Frijol, R. Soltero 560 (IBUG); Orillas del pueblo de Pihuamo, Arreola-N. s.n. (IBUG); TECOLOTLAN, Rancho el Saucillo, Puerta Pesada, García-R. y Corona-O.1578A (IBUG); Tamazulita, La Ciénega, Corona-O. y Vargas-P. 9 (IBUG*); TOLIMÁN, Brecha San Pedro Toxín, Arreola-N. et al. 1177 (IBUG); ZAPOTITLÁN DE VADILLO, La Parotilla, García-R. et al.1582 (IBUG*); MICHOACÁN, COALCOMÁN, 57 kilómetros entre Coalcomán y Villa Victoria, S. Arias 822 (MEXU); LOS REYES, Barranca de Los Chorros del Varal, M. Chazaro y A. García 7334 (MEXU); TZITZIO, Entrada a Tzitzio, García-R. et al. 1649 (IBUG*); Guanajuatito, García-R. et al. 1650 (IBUG*); El Reparo, García-R. et al. 1652 (IBUG*); El Devanador de Rivera, García-R. et al. 1653 (IBUG*); 5 km después de El Devanador de Rivera, García-R. et al. 1655 (IBUG*); ZACAPU, Cañada Barranca Honda, García-R. et al.1662 (IBUG*); ZINAPÉCUARO, El Zapote, García-Aguilar 34 (CHAPA).

CULTIVADOS. GUERRERO, ACAPULCO, Parque nacional El Veladero, Cerro Carabali, N Acosta 649 (IEB); JALISCO, AMACUECA, San Juanito, Agua Caliente; Corona-O. y Vargas-P.61 (IBUG*); Vicente Guerrero, García-R. et al.; 1642, (IBUG*); El Coral, García-R. y Meza-C.1632 (IBUG, IBUG* MEXU, ENCB); El Pedregal, García-R. y Meza-C.1625 (IBUG*); ATOYAC, Poblado de San Juan, García-R. y Corona-O.1607 (IBUG, IBUG* MEXU, ENCB); AUTLÁN, Cerca del Corcovado, carretera Guadalajara-Autlán 12 km antes de llegar a Autlán, L.M.V. de Puga s.n. (IBUG); Poblado de Ahuacapán, Arreola-N. et al. 119 (IBUG); CUAUTITLÁN DE GARCÍA BARRAGÁN, 28 Km al NE de Cuautitlán, camino a Ayotitlán, por la brecha Cuautitlán-Ayotitlán, Arreola-N. 693 (IBUG); SAYULA, Potrero La Huizachera, García-R. y Corona-O. 1581 (IBUG, IBUG* MEXU, ENCB), Potrero La Huizachera, Corona-O. 54 (IBUG*); TENAMAXTLÁN, Tenamaxtlán, Arreola-N. y R. Soltero 124 (IBUG); ZACOALCO, Barranca de los Laureles, Corona-O. y Vargas-P. 44 (IBUG*); Rancho la Cruz, Corona-O. y Vargas-P. 40 (IBUG*); MICHOACÁN, CHINICUILA, km 58.7 de la brecha que va de Coalcomán a Aquila, H. Sánchez 4256 (MEXU); HUETAMO, Quechendio, García-R. et al. 1658 (IBUG*); LA PIEDAD, Bravo-Hollis s.n. 4072 (MEXU); NOCUPETARO, El Zapote, J. Andrés s.n. (MEXU); TIQUICHEO, Las Mojarras, García-R. et al. 1657 (IBUG*); Los Cuitaces, García-R. et al. 1659 (IBUG*); TZITZIO, Palmarito, García-R. et al. 1648 (IBUG*).

CONDICION DESCONOCIDA. ESTADO DE MÉXICO, TLATLAYA, Piedra Parada, San Antonio del Rosario, E. Matuda 5030 (MEXU); JALISCO, AUTLÁN, Las Paredes, M. Hernández s.n. (IBUG); EL LIMÓN, Ejido el Realito, J. Durán s.n. (IBUG); MICHOACÁN, CUAHUAYANA, Cuahuayana, cerca del mar, L. Scheinvar 1627 (ENCB); MORELOS, COATLÁN DEL RIO, Grutas de Cacahuamilpa, J. Vázquez 3229 (MEXU); VERACRUZ, JALCOMULCO, Jalcomulco, Cerro del brujo, 3 km al N de Jalcomulco, C. Campos y P. Zamora 8661 (XAL).

\section{Hylocereus purpusii (Weing.) Britton \& Rose}

SILVESTRES. COLIMA, TECOMÁN, L. Scheinvar 1629 (MEXU); GUERRERO, ACAPULCO, La Venta (Ambrosio Figueroa); presa frente a la desenvocadura del Rio Papagayo, en el Rio Omitlán, H. Kruse 3020 (MEXU); CHILPANCINGO, Sierra de Acayuizotla, L. Scheinvar 5335 (MEXU); JUAN ESCUDERO, Tierra Colorada, H. Kruse $3020 b$ (MEXU); Britton y Rose 3020 (MEXU); $10 \mathrm{~km}$ al sur carretera Ayutla, H. Kruse 2935 (MEXU); $1 \mathrm{~km}$ al sur de Tierra Colorada, Bravo-Hollis s.n. (MEXU); JALISCO, AMACUECA, La Huázima, García-R. y Meza-C.1623 (IBUG, IBUG* MEXU, ENCB); Vicente Guerrero, García-R. y Meza-C.1621 (IBUG*); ATOYAC, Isla Grande, Laguna de Sayula, García-R. y Meza-C. 1630 (IBUG*); Arreola-N. et al. 1368 (IBUG); Agua Azul, García-R. y Meza-C. 1641 (IBUG, IBUG* MEXU, ENCB); Poblado de San Juan; García-R. y Vargas-P. 1598 (IBUG, IBUG* MEXU, ENCB); CABO CORRIENTES, km 13 de la carretera El Tituito-Tehuamixtle, M. Martínez y A. Rodríguez 254 (IBUG); CUAUTITLÁN DE GARCÍA BARRAGÁN, Ayotitlán, base sur de la Sierra de Manantlán, R. Guzmán 7010 (IBUG); EJUTLA, Junto a la presa, CoronaO. y Vargas-P. 18 (IBUG*); El Salto, 2-3 km al SE del poblado de Tequesquitlán, L. Guzmán et al. 28 (IBUG); HOSTOTIPAQUILLO, Puente Barranquitas, por la ribera del rio, L. Guzmán y A. Flores 83 (IBUG); IXTLAHUACÁN DE LOS MEMBRILLOS, Barranca 
Distribución de HyLOCEREUS EN MÉXICO

Apéndice 1. Continuación

Húmeda de Santa Rosa, L.M.V. de Puga y S. Carvajal 9784 (MEXU); LA HUERTA, Brecha al rancho Cuixmala a $2 \mathrm{~km}$ del entronque con la carretera Barra de Navidad-Pto. Vallarta, Arreola-N. et al. 157 (IBUG); PUERTO VALLARTA, $2 \mathrm{~km}$ al W del poblado de Las Palmas, Arreola-N. et al. 160 (IBUG, ENCB); TECOLOTLAN, Tamazulita, La Ciénega, Corona-O. y Vargas-P. 6 (IBUG*); MICHOACÁN, LA HUACANA, Pedregal del Volcán Jorullo, Bravo-Hollis 5528 (MEXU); TACÁMBARO; Tacámbaro-Jorullo, $L$. Scheinvar 1654 (MEXU); TZITZIO, Guanajuatito, cañada encinar, García-R. et al. 1651 (IBUG*); 5 km después de El Devanador de Rivera, García-R. et al. 1654 (IBUG*); Los Ciruelos, García-R. et al. 1656 (IBUG*); TURICATO, Barranca del Caramicuas, García-R. et al. 1660 (IBUG*); ZACAPU, Cañada Barranca Honda, García-R. et al. 1661 (IBUG*); Barranca Honda, García-R. et al. 1663 (IBUG*); 6 km después de Barranca Honda, García-R. et al. 1664 (IBUG*); Hacia el Coyolote, García-R. et al. 1665 (IBUG*); Carretera a Zacapu, García-R. et al. 1666 (IBUG*); NAYARIT, ACAPONETA, Hacienda de Mariquita, Corona-O. et al. 97 (IBUG*); AHUACATLÁN, Malpaís del Volcán Ceboruco, sobre la carretera Ahuacatlán-Tepic, cerca de Copales, M. Cházaro-B. et al. 6951 (MEXU, IEB, XAL); El Marquesado, Corona-O. et al. 110 (IBUG*); Corona-O. et al. 111 (IBUG*); AMATLAN DE LAS CAÑAS, Corona-O. et al. 77 (IBUG*); Balneario El Manto, ca. $1.5 \mathrm{~km}$ al O de El Rosario, Carrillo-Reyes y D. Cabrera 7023 (IBUG*); El Rosario, Corona-O. et al. 75 (IBUG*); El Rosario, Corona-O. et al. 76 (IBUG*); DEL NAYAR, Vereda de la Mesa del Nayar al Cangrejo, que cruza por la barranca, R. Ramírez 559 (MEXU); Potrero de la Palmita, a $10 \mathrm{~km}$ al E de la cortina de la P. H. Aguamilpa, G. Franco et al. 2725 (MEXU); Lado E de la Luna, Santa Teresa del Oro, E. Greenwood 5321 (MEXU); Presa San Rafael, Puente Xicuixtle, García-R. et al. 1645 (IBUG*); Texcalame, Corona-O. et al. 94 (IBUG*);Texcalame, Corona-O. et al. 95 (IBUG*); HUAJICORI, Agua Caliente, Corona-O. et al. 99 (IBUG*); JALA, Volcán Ceboruco, Garcia-R. y Vargas-P. 1608 (IBUG*); El Capulin, García-R. et al. 1646 (IBUG*); Puerta de Jala la vieja, García-R. et al. 1647 (IBUG*); Volcán Ceboruco, García-R. y Corona-O.1589 (IBUG, IBUG* MEXU, ENCB); Jomulco, Corona-O. et al. 74 (IBUG*); JALCOTOTAN, Corona-O. et al. 108 (IBUG*); Corona-O. et al. 109 (IBUG*); POCHOTITLÁN, 20 km al E de Puga, por camino a Pochotitlán, cerca de las faldas de la serranía al N del camino Puga-Pochotitlán, O. Téllez-B. 11309 (MEXU); ROSA MORADA, San Juan Bautista, Corona-O. et al. 96 (IBUG*); RUÍZ, Corona-O. et al. 91 (IBUG*); Corona-O. et al. 92 (IBUG*); El Zopilote, Corona-O. et al. 93 (IBUG*); $7 \mathrm{~km}$ al W del Venado, camino a San Pedro Ixcatán y el Zopilote, O. Téllez y G. Flores 11825 (MEXU); SAN BLAS, La Tobara, O. Téllez 12496 (MEXU); SAN PEDRO LAGUNILLAS, Arroyo El Guayabo, Ejido Cerro Pelón; García-R. y Vargas-P. 1610 (IBUG*); SANTA MARÍA DEL ORO, Laguna de Santa María del oro, Arreola-N. y R. Ramírez s.n. (IBUG*);TEPIC, Km 655 Carretera Guadalajara-Tepic, Bravo-Hollis 233 (MEXU); La Herradura, Corona-O. et al. 78 (IBUG*); Francisco I. Madero, Colonia la pareja, Corona-O. et al. 83 (IBUG*); San Francisco, Corona-O. et al. 87 (IBUG*); San Rafael, Corona-O. et al. 88 (IBUG*); San Felipe, Corona-O. et al. 89 (IBUG*); TUXPAN, Tuxpan, sobre pedregales, Bravo-Hollis s.n. (MEXU); OAXACA, OAXACA, Parte media del cerro San Felipe, al N de la ciudad de Oaxaca, entrando por el vivero forestal, H. Hernández 2564 (MEXU); SAN JUAN BAUTISTA CUICATLÁN, Cerro Picacho, a orillas de Rio Grande, San José El Chilar, E. Cruz 1011 (MEXU); 6 km al NE de Santiago Quiotepec, P. Tenorio 17415 (MEXU); QUERÉTARO, CADEREYTA, Carricillo, L. Scheinvar et al. 4340 (MEXU); SAN LUIS POTOSÍ, TAMAZUNCHALE, Carretera 85, a 39 minutos al N de Tamazunchale, D. Dunn et al. 23105 (ENCB); SINALOA, CONCORDIA, La concordia, Corona-O. et al. 106 (IBUG*); Zavala, Corona-O. et al. 107 (IBUG*); CULIACÁN, Tepuche, Molo Viejo, Corona-O. et al. 104 (IBUG*); ESCUINAPA, El Camarón, Corona-O. et al. 101 (IBUG*).

CULTIVADOS. DISTRITO FEDERAL, Delegación Coyoacán, Jardín Botánico Exterior, Cd. Universitaria, L. Scheinvar 3166 (MEXU); ESTADO DE MÉXICO, TEMAZCALAPA, Pueblo San Mateo Teopancala, convento franciscano del siglo XVI, L. Scheinvar 3387 A (MEXU); JALISCO, AMACUECA, San Juanito, Agua Caliente; García-R. y Vargas-P. 1590 (IBUG, IBUG* MEXU, ENCB); El Piojo, García-R. y Meza-C.1620 (IBUG*); El Coral, García-R. y Meza-C.1622 (IBUG, IBUG* MEXU, ENCB); El Pedregal, García-R. y Meza-C.1624 (IBUG*); Los Nogalitos, García-R. y Meza-C.1638 (IBUG*); Tepec, García-R. y Meza-C.1640 (IBUG*); AUTLÁN, El Corcovado, antes de llegar a Autlán, km 139 carretera Guadalajara-Barra de Navidad, Arreola-N. et al. 539 (IBUG); PUERTO VALLARTA, Los Arcos de Puerto Vallarta, Cochrane et al. 13083 (IBUG); SAYULA, Potrero La Huizachera, García-R. y CoronaO.1584 (IBUG, IBUG* MEXU, ENCB); Corona-O. y Vargas-P. 53 (IBUG*); TECHALUTA DE MONTENEGRO, El Zapote, García-R. y Meza-C. 1637 (IBUG*); ZACOALCO, Rancho la Cruz, Corona-O. y Vargas-P. 39 (IBUG*); Zacoalco, Barranca de los Laureles, Corona-O. y Vargas-P. 45 (IBUG*); ZAPOTITLÁN DE VADILLO, Santa Elena, García-R. et al.1583 (IBUG*); MICHOACÁN, ARTEAGA, Patacuas, García-R. et al. 1667 (IBUG*); Carretera a Arteaga, García-R. et al. 1668 (IBUG*); Los Amates, carretera a Arteaga, García-R. et al. 1669 (IBUG*); NAYARIT, HUAJICORI, Corral de piedra, Corona-O. et al. 98 (IBUG*); Huajicori, Corona-O. et al. 100 (IBUG*); ROSA MORADA, San Juan Bautista, Corona-O. et al. 112 (IBUG*); RUIZ, El venado, Corona-O. et al. 90 (IBUG*); SAN BLAS, Casa de la señora Zoila Maravilla, Corona-O. et al. 79 (IBUG*); Singayta, Corona-O. et al. 80 (IBUG*); Ejido la palma, Corona-O. et al. 81 (IBUG*); San Blas, Corona-O. et al. 82 (IBUG*); TEPIC, Santiago Pochotitan, Corona-O. et al. 85 (IBUG*); Corona-O. et al. 86 (IBUG*); OAXACA, SAN PEDRO POCHUTLA, Puente Los Azufres, carretera Oaxaca-Pochutla, cerca de Chacalapa, H. Sánchez 2316 (MEXU); QUERÉTARO, EZEQUIEL MONTES, Jahuey Grande, L. Scheinvar et al. 4340a 
LuZ Adriana García-Rubio et AL.

Apéndice 1. Continuación

(MEXU); SINALOA, CULIACAN, No se cita, Corona-O. et al. 102 (IBUG*); Tepuche, Las Juntas, Corona-O. et al. 103 (IBUG*); San Francisco de Tacuichamona, Corona-O. et al. 105 (IBUG*); MAZATLÁN, 46 Millas al E de Mazatlán, por la carretera a Durango, Kimnach y Moran 94 (MEXU).

CONDICION DESCONOCIDA. JALISCO, JUCHITLÁN, P. Mejía s.n. (IBUG).

\section{Hylocereus undatus (Haw.) Britton \& Rose}

SILVESTRES. CAMPECHE, CALAKMUL, $65 \mathrm{~km}$ al S de Conhuas en el centro ceremonial Calakmul, limite N del Petén Guatemaltéco, E. Cabrera et al. 4411 (MEXU); Ejido Narciso Mendóza. A 1 km del poblado, carretera Xpujil-Narciso, D. Alvares 319 (MEXU); CHAMPOTÓN, Laguna Noh, C. Chan y E. Ucán 959 (CICY); ESCARCEGA, Laguna de Silvituc, Bravo-Hollis 2680 (MEXU); XPUJIL, Km 3.7 Xpujil-Dzibalchén Cálix de Dios y R. Castillo 588 (CICY); CHIAPAS, CINTALAPA, 22.5 km al NE Tepanatepec, rumbo a Tuxtla Gutierrez. Reseva de la Biósfera La Sepultura, Gómez-H.et al. 2186 (MEXU); 2.6 km al NW de la Hacienda Monserrate, rumbo a Betel. Monserrat se encuentra a 15 km al NW de Vista Hermosa, Gómez-H.y Hernández 2321 (MEXU); CHAPA DE CORZO, Puente Belisario Domínguez, Greenwood s.n. (MEXU); El Sumidero, cerca de Tuxtla Gutiérrez, Bravo-Hollis s.n. (MEXU); CHICOASÉN, Mirador Manos que Imploran, ca. 20 km al NE de San Fernando, rumbo a Chicoasén, Gómez-H. et al. 2197 (MEXU); ESCUINTLA, Esperanza, E. Matuda 17771 (MEXU); MOTOZINTLA DE MENDOZA, Ca. 2 km al NW de Motozintla sobre terraceria al Carrizal, Gómez-H. y A. Cervantes 2085 (MEXU); 1 km al NE de Motozintla, rumbo a Mazapa, Gómez-H. et al.2191 (MEXU); Belisario Domínguez, a los lados del rio. Belisario Domínguez se encuentra a 30 km al SW de N de Motozintla, Gómez-H. y A. Cervantes 2082 (MEXU); Ca. 2 km al NW de Motozintla sobre terraceria al Carrizal, Gómez-H. y A. Cervantes 2086 (MEXU); OCOSINGO, Terán, 1 km al N de Emiliano Zapata, S. Arias y U. Guzmán 1020 (MEXU); OCOZOCOAUTLA DE ESPINOZA, 6 millas al Este de Ocozocoautla, Carretera 190 a México, Breedlove 10007 (ENCB); Rancho La Victoria, García-R. et al. 1677 (IBUG*); SUCHIAPA, Km 13.5 carretera a Suchiapan, cerca al rancho La Habana, García-R. et al. 1678 (IBUG*); La Ciénega, Suchiapan, García-R. et al. 1679 (IBUG*); Entrada a Aeropuerto, carretera a Suchiapan, Ramírez-M. y Cruz-H. 855 (IBUG*); 1 km de la Finca San Angel, Ramírez-M. y Cruz-H. 858 (IBUG*); VILLAFLORES, Guadalupe Victoria, García-R. et al. 1680 (IBUG*); PARRAL, Crucero de Jericó, García-R. et al. 1681 (IBUG*); SAN FERNANDO, El Barrancón, a un lado de La Encañada, que se encuentra a 13 km al NW de Tuxtla Gutiérrez por el camino a San Fernando, Gómez-H. 2094 (MEXU); La Encañada, 1 km delante de La Virgen, rumbo a San Fernando. La Encañada se encuentra a 13 km al NW de Tuxtla Gutiérrez, Gómez-H. et al. 2098 (MEXU); TONALÁ, Entre Arriaga y Tonalá, Bravo-Hollis 5221 (MEXU); ZINACATLÁN, 15 km después de San Cristobal rumbo a Tuxtla Gutiérrez, A. Espejo 1693 (ENCB); TRINITARIA, Puente Rio San Juan, García-R. et al. 1691 (IBUG*); ESTADO DE MÉXICO, EL ORO, Entre el Oro y Villa Victoria, E. Matuda et al. 30930 (MEXU); NEPANTLA, E. Matuda et al. 28374 (MEXU); TLALMANALCO, San Rafael y cercanías, ladera húmeda, E. Matuda et al. 30907 (MEXU); GUANAJUATO, ACÁMBARO, 3 km al NE de El Sauce, A. Rubio 570 (ENCB); ATARJEA, Cañón de Atarjea, ca. 1.5 a 2 hrs., de camino a pie sobre el lecho del rio R. Bárcenas 891 (MEXU, IEB); Cañón de Atarjea, ca. 2 hrs caminando sobre el lecho del rio. Gómez-H. y R. Bárcenas 466 (MEXU); YURIRIA, San Francisco de la Cruz, camino Valle de Santiago-Yuriria, P. Colunga, 14 (MEXU, ENCB); GUERRERO, CHILPANCINGO DE LOS BRAVO, Parte alta de la cañada de Acahuizotla, H. S. M. U. G. et S. A. M. 3941 (MEXU); MOCHITLÁN, Cajales, H. Kruse 3014 (MEXU); JALTOCAN, Revolución Mexicana, Ramírez-M. y Cruz-H. 39 (UACH); HIDALGO, ACOXCATLÁN de Guerrero Bachillerato, Ramírez-M. y Cruz-H. 38 (UACH); CHAPULHUACAN, Neblinas, Ramírez-M. y Cruz-H. 40 (UACH); HUEJUTLA DE LOS REYES, Coxhuaco, A 3 km de Huejutla, L. Scheinvar 2314 (MEXU); JALTOCAN, Revolución Mexicana, Ramírez-M. y Cruz-H. 39 (UACH); METZTITLÁN, Barranca de Meztitlán, Bravo-Hollis 3360 (MEXU); Desviación al Rio Metztitlán, Las Pilas, L. Scheinvar et al. 3413 (MEXU); YEHUALICA, El Crucero, Ramírez-M. y Cruz-H. 37 (UACH); JALISCO, ATOYAC, Isla Grande. Laguna de Sayula, Arreola-N. et al. 1369 (IBUG); Laguna de Sayula, Isla Grande, parte alta, E. Villegas. y M. Macías 494 (IBUG); TAMAZULA, López s.n. (MEXU); MICHOACÁN, ARTEAGA, Rancho La Parota, Las Juntas, cerca del Infiernillo, L. Scheinvar 2163 (MEXU); NAYARIT, TEPIC, Mirador del Águila, $19 \mathrm{~km}$ al NW de Tepic por la carretera vieja a Mazatlán, Gómez-H. 1995 (MEXU); OAXACA, ASUNCIÓN IXTALTEPEC, $1.0 \mathrm{~km}$ en línea recta al NE (32) de Nizanda, E. Pérez et al. 1132 (MEXU); GUEVEA DE HUMBOLD, Cabecera Municipal, Ramírez-M. et al. s.n. (IBUG*); MIAHUATLAN, El Tunillo, Corona-O. et al. 150 (IBUG*); PINOTEPA DE DON LUIS, Federal 125 de Pinotepa-Putla, antes de Ilegar a Zacatepec, T. Hernández 6 (MEXU); PUTLA VILLA DE GUERRERO, Ca. 4 km al NE de San Pedro Siniyuvi, E. Solano 428 (MEXU, CHAPA); SAN CARLOS YAUTEPEC, San Miguel Chongo, E. Martínez 32112 (MEXU); SAN JUAN BAUTISTA CUICATLÁN, Barranca cuatima a 200 m de cuatima, R. García 928 (MEXU); Buena Vista, brecha entre Quiotepec y Coyula, R. García 645 (MEXU); SAN JUAN DE LOS CUES, $2.5 \mathrm{~km}$ al NE de San Juan de los Cues, colecta alrededor de la presa, A. Salina et al. 4113 (MEXU); SAN MIGUEL CHIMALAPA, Arroyo Hierba Santa, en las faldas del Cerro Guayabitos, que desemboca al rio escondido entre Bento Juárez y Col. Díaz Ordáz, ca. 38 km en linea recta al N de San Pedro Tapanatepec, J. Salomón 1509 (MEXU); SAN MIGUEL DEL PUERTO, 
Distribución de Hylocereus en MéXico

Apéndice 1. Continuación

Finca Montecarlo, 500m al N por el camino de la zanja, J. Rivera 2514 (MEXU); Finca Montecarlo, 600 m, A. Saynes et al. 2182 (MEXU); El Enjambre camino a la Constancia, J. Pascual 1530 (MEXU); Cerro Campana, J. Pascual 1512 (MEXU); SAN MIGUEL TLACAMAMA, 2 km al NW de la Catalina o $18 \mathrm{~km}$ al NW de Pinotepa, R. Torres et al. 5480 (MEXU); SAN PEDRO TAPANATEPEC, 22 km al NE de Tapanatepec, Gómez-H. 2069 (MEXU); SAN PEDRO TOTOLAPAN, San Juan Guegoyachi, Ramírez-M. et al. s.n. (IBUG*); SANTO DOMINGO TEHUANTEPEC, Dto. Tehuantepec, El Carrizal o la Cañada, camino Limón Carrizal. El Limón esta a 17 km al O de Tehuantepec, entrando por hierba Santa, R. Torres et al. 9828 (MEXU); SANTO DOMINGO ZANATEPEC, Rancho El Zapote, Ramírez-H. et al. s.n. (IBUG*); YAUTEPEC, Carretera de San Juan Guegotachi a Jactepec, Ramírez-M. et al. s.n. (IBUG*); Carretera San Bartolo Yautepec a Santa Maria Quiegolani, Ramírez-H. et al. s.n. (IBUG*); Carretera de San Juan Guegotachi a Jactepec, Corona-O. et al. 131 (IBUG*); Carretera de San Juan Guegotachi a Jactepec, Corona-O. et al. 133 (IBUG*); PUEBLA, CUAUTEMPAN, San Pedro Huaytentan, Ramírez-M. y Cruz-H. 25 (UACH); FILOMENO MATA, San Pedro Tlaolantongo, Ramírez-M. y Cruz-H. 27 (UACH); FRANCISCO Z. MENA, Ojo de Agua, Ramírez-M. y Cruz-H. 30 (UACH); HUEYTAMALCO, Teypactipan, Ramírez-M. y Cruz-H. 23 (UACH); TENANPULCO, Camino a Jicarillas, Ramírez-M. y Cruz-H. 26 (UACH); VENUSTIANO CARRANZA, El Búfalo, Ramírez-M. y Cruz-H. 29 (UACH); XICOTEPEC, San Lorenzo, Ramírez-M. y Cruz-H. 28 (UACH); QUERÉTARO, JALPAN DE SERRA, El Trapíche, al N de Jalpan, L. Scheinvar y C. Rodríguez 4666 (MEXU); Puente Conka, B. Rosales y O. Guerrero 4 (MEXU); La Canastilla, al oriente de Emiliano Zapata S.L.P., López-L. 183 (IEB); Puerto de Animas, RamírezM. y Cruz-H. 45 (CHAPA); LANDA DE MATAMOROS, Rio Moctezuma, 15 km al Sureste de Agua Zarca, H. Rubio 2410 (MEXU); Entre el Lobo y Agua Zarca, L. Scheinvar et al. 5101 (MEXU); La Polvareda, Ramírez-M. y Cruz-H. 44 (CHAPA); PINAL DE AMOLES, Jalpan, $0.5 \mathrm{~km}$ al S de Escanelilla, L. Scheinvar et al. 4040 (MEXU); SAN JUAN DEL RIO, Cerro Gordo, $8 \mathrm{~km}$ al SE de la cabecera municipal de San Juan del Rio, F. Rodríguez y M. Osorno 1 (CHAPA); QUINTANA ROO, FELIPE CARRILLO PUERTO, Medina y Cetzal 103 (CICY); SAN LUIS POTOSÍ, AQUISMÓN, 25 km al W de Ciudad Valles, sobre la carretera a Rio Verde, Rzedowski 24424a (ENCB); AXTLA DE TERRAZAS, Aguacatita, 3km al N de Tamazunchale, García-R. y Valdívia-M. 1611 (IBUG); EL NARANJO, 13 km al N del El Naranjo, Hernández-X. 7667 (MEXU); TAMAZUNCHALE, Rio Axtla, L. Scheinvar et al. 3426 (MEXU); Km 2.5 a Tanquian, Ramírez-M. y Cruz-H. 41 (UACH); TAMPAMOLON, San José, Ramírez-M. y Cruz-H. 42 (UACH); XILITLA, San Rafael, Ramírez-M. y Cruz-H. 43 (UACH); TABASCO, BALANCAN, El Zapote, Ramírez-M. y Cruz-H. 2 (UACH); HUIMANGUILLO, Villaflores, Ramírez-M. y Cruz-H. 8 (UACH); Pejelagarto, Ramírez-M. y Cruz-H. 9; JONUTA, Rancho El Pastal, a 3 km hacia Cd. Pemex de la desviación a Monte Grande, M.A. Guadarrama et al. 6845 (MEXU); MACUSPANA, Pitahaya, Ramírez-M. y Cruz-H. 3; Montelargo, Ramírez-M. y Cruz-H. 4 (UACH); NACAJUCA, Jaloya, Ramírez-M. y Cruz-H. 6 (UACH); PARAISO, Muctezuma, Ramírez-M. y Cruz-H. 7 (UACH); TEAPA, Teapa, Ramírez-M. y Cruz-H. 5; TENOSIQUE, Ejido Corregidora Ortíz de Mezcalapa, R. Escolastico 183 (ENCB); Emiliano Zapata, Ramírez-M. y Cruz-H. 1 (UACH); VILLA HERMOSA, Villa Hermosa, L. Scheinvar 2304 (MEXU); VERACRUZ, ACAYUCAN, 3 km delante de Acayucan por la carretera libre a Coatzacoalcos, L. Scheinvar et al. 6052 (MEXU); Comejen, Ramírez-M. y Cruz-H. 11 (UACH); ACTOPAN, Cerro El Pailón (Sierra Manuel Díaz); R. Acosta et al. 742 (MEXU, XAL); Entre Actopan y Trapiche del Rosario, L. Scheinvar et al. 866 (MEXU); ALVARADO, A 23 km de Alvarado rumbo a Veracruz La Piedra A. Lot 1394 (MEXU); 2.8 km después de Salinas, rumbo a Veracruz, carretera AlvaradoVeracruz, A. Espejo et. al. 7016 (XAL); BENITO JUAREZ, El Paraje, Ramírez-M. y Cruz-H. 34 (UACH); CATEMACO, Laguna, en una isla, L. Scheinvar 6067 (MEXU); Orilla N de la Laguna de Catemaco, hacia Zontecomapa, L. Scheinvar 647 (MEXU); Teotepec, Ramírez-M. y Cruz-H. 12 (UACH); CÓRDOBA, 3 km delante de Cuitlahuac, L. Scheinvar 6050 (MEXU); COSAMALOAPAN, Chicozapote, Ramírez-M. y Cruz-H. 15 (UACH); CUITLAHUAC, Rastro de Cuitlahuac, Ramírez-M. y Cruz-H. 17 (UACH); EL HIGO, El Higo, Ramírez-M. y Cruz-H. 36 (UACH); EMILIANO ZAPATA, Cerca de Plan del Rio y Rinconada, C. Delgadillo 13 (MEXU); IXHUATLÁN DE MADERO, V. Smith 123-B (XAL); JALCOMULCO, 2 km al NE de Jalcomulco, G. Castillo 18015 (MEXU, IEB); (XAL); JALTIPAN, 4 k delante de Lomas de Techa, camino a Hidalgotitlán, L. Scheinvar et al. 6057 (MEXU); JUCHIQUE DE FERRER, La Pila, Ramírez-M. y Cruz-H. 21 (UACH); LA ANTIGUA, Barranca de Panoaya, 2 km al NO de El Coyol, M. Medina. y R. Acosta 173 (MEXU, XAL); MARTíNEZ DE LA TORRE, El Refugio, Ramírez-M. y Cruz-H. 22 (UACH); MEDELLÍN, Entre paso del Toro y Alvarado, Bravo-Hollis s.n. (MEXU); 13 Km al SE Paso del Toro, B. Leuenberger y C. Schiers 2500 (MEXU); MISANTLA, Cerro Quebrado, entre Misantla y Martínez de la Torre, R. Riba 270 (MEXU); NAOLINCO, 3 km después de la Concepción rumbo a San Pablo, R. Ortega 338 (XAL); PÁNUCO, Topila ranchitos. Base del cerro, C. Gutiérrez y E. Montoya 1853 (XAL); PAPANTLA DE OLARTE, $2.5 \mathrm{~km}$ al E entrando a las ruinas de El Tajín, 4km SW de Papantla, M. Nee y G. Diggs 24628 (XAL); El Chote, Ramírez-M. y Cruz-H. 24 (UACH); POZA RICA, 1 km al NE de Tihuatlan, Bruce et al. 1762 (MEXU); PUENTE NACIONAL, Entre Puente Nacional y el Crucero, M. Cházaro y L. Robles 2885 (MEXU); Hato de la Higuera, M. Sousa 2718 (MEXU); SAYULA DE ALEMÁN, Los Reyes, Ramírez-M. y Cruz-H. 10 (UACH); SAN ANDRÉS TUXTLA, Playa Balzapote; ca. 4 km al NE de la Estación de Biología de Los Tuxtlas, Gómez-H. et al. 2147 (MEXU); SANTIAGO TUXTLA, Rancho El Zapote, Ramírez-M. y Cruz-H. 13 (UACH); TANTOYUCAN, Sabana Grande, Ramírez-M. y Cruz-H. 33 (UACH); El Rincon, Ramírez-M. y Cruz-H. 35 (UACH); TE- 
LuZ Adriana García-Rubio et AL.

Apéndice 1. Continuación

MAPACHE, $4 \mathrm{~m}$ al SE de Potrero del Llano, Bruce et al. 1773 (MEXU); TEPETZINTLA, Ejido El Pueblo, Ramírez-M. y Cruz-H. 32 (UACH); TIHUATLAN, Tecomate, Ramírez-M. y Cruz-H. 31 (UACH); TLALIXCOYAN, Piedras Negras, Ramírez-M. y Cruz-H. 16 (UACH); VEGA DE ALATORRE, Cumbre del cerro Chichimecas, B. Guerrero y J. Calzada 1910 (XAL); Ca. De 9km al SW de Vega de Alatorre, rumbo a Yecuatla, Gómez-H. 2262 (MEXU); VILLA JOSÉ AZUETA, Ejido Arrollo de la Cruz, Ramírez-M. y Cruz-H. 14 (UACH); XICO, San Marcos Xico, Ramírez-M. y Cruz-H. 20 (UACH); ZONTLA, El Guaje, Ramírez-M. y Cruz-H. 19 (UACH); XALAPA, Camino al Sumidero, M. Zola 828 (XAL); YUCATÁN, DZONCAUICH, Chacmay $2 \mathrm{~km}$ al E Dzoncauich, Puch-A. y YamP. 1374 (CICY); OXKUTZCAB, Xul, camino a milpas rumbo este, O. Sanabria y P. Simá 399 (MEXU); Xul, D. Zizumbo et al. 1065 (XAL); PROGRESO, Isla Pérez, Arrecife Alacranes, sin colector, s.n. (CICY); RIO LAGARTOS, 8 km al E Crucero Rio Lagartos, rumbo al Rancho San Salvador, sin colector, s.n. (CICY); SAN FELIPE, San Felipe a Rio Lagartos, Granados et al. 25 (CICY); TICUL, Carretera Ticul-Santa Elena, N. Narváez y A. Puch 584 (XAL), Santa Elena, N. Narváez y A. Puch 542 (CICY).

CUltivadOS. COLIMA, COLIMA, Ciudad de Colima, F.J. Santana y Arreola-N. s.n. (IBUG); CHIAPAS, ACALA, Las Palomas, García-R. et al. 168 (IBUG*); BERRIOZABAL, San Antonio Bombano, García-R. et al. 1676 (IBUG*); San Antonio Bombano, Ramírez-M. y Cruz-H.850 (UACH); El Capricho, Ramírez-M. y Cruz-H.848 (UACH); CHAPA DE CORZO, Salvador Urbina, García-R. et al. 1683 (IBUG*); Rivera Cabeza de Agua, Ramírez-M. y Cruz-H.860 (UACH); COMITÁN DE DOMÍNGUEZ, Tzimol, García-R. et al. 1689 (IBUG*); Rio Grande, Ramírez-M. y Cruz-H.868 (UACH); FRONTERA COMALAPA, San Caralampio, García-R. et al. 1692 (IBUG*); Jaboncillo, García-R. et al. 1694 (IBUG*); Santa Martha, Ramírez-M. y Cruz-H.874 (UACH); MAPASTEPEC, Camino Roberto Barrios al Castaño, Reserva La Encrucijada, F. Hernández 358 (MEXU); SOCOLTENANGO, El Banco, Ramírez-M. y CruzH.866 (UACH); TRINITARIA, Delicias, Ramírez-M. y Cruz-H.870 (UACH); Entronque Rodulfo Figueroa, Ramírez-M. y Cruz-H.872 (UACH); TUXTLA GUTIERREZ, Copoya, Ramírez-M. y Cruz-H. 852 (UACH); VENUSTIANO CARRANZA, Colonia El Puy, GarcíaR. et al. 1687 (IBUG*); Venustiano Carranza, entrada principal, Ramírez-M. y Cruz-H.864 (UACH); VILLAFLORES, Agronomos mexicanos, García-R. et al. 1682 (IBUG*); VILLA COMALTITLÁN, Emiliano Zapata, Entrada al Cerrito, Ramírez-M. y Cruz-H.862 (UACH); ESTADO DE MÉXICO, Coacalco, Av. 3 Calle 14, SW esquina, Col. San José Ejidal, R. Bye y E. Linares 28910 (MEXU); GUANAJUATO, YURIRIA, San Miguelito el Alto, L. Scheinvar 3450 (MEXU); San Andrés Enguaro, L. Scheinvar 3443 (MEXU); GUERRERO, CHILPANCINGO, Rincón Viejo, H. Kruse 2580 (MEXU); HIDALGO, CALNALI, No se cita, Gómez-H. 10 (MEXU); METZTITLÁN, Bravo-Hollis 5930 (MEXU); JALISCO, GUADALAJARA, Hacienda de Portillo, Barranca de Huentitán, R. Acevedo et al. 1671 (IBUG); Huerto familiar, L. Portillo s.n. (IBUG); Huerto familiar, casa particular, J. Suárez s.n. (IBUG); Huerto familiar, casa particular, S. Beiza s.n. (IBUG); SAYULA, Potrero La Huizachera, Garcia-R. y Corona-O. 1588 (IBUG, IBUG* MEXU, ENCB); TÉNAMAXTLÁN, Rancho Miraplanes, Arreola-N. y R. Soltero 127 (IBUG); La Cofradía de los Coyotes, 2km al S. De Tenamaxtlán, Arreola-N. y R. Soltero 126 (IBUG); ZACOALCO DE TORRES, Huerto familiar de la Sra. Refugio Castillo, población de Zacoalco, Arreola-N. et al. 1453 (IBUG); ZAPÓPAN, Casa situada en Av. C y calle 3, colonia Seattle, M. Chazaro y P. Hernández 7719a (IBUG); ZAPOPAN, Cultivado en el Jardín Botánico del Instituto de Botánica (IBUG) Universidad de Guadalajara, Arreola-N. s.n. (IBUG); MORELOS, Tepoztlán, Tepoztlán, L. Scheinvar 2172 (CHAPA); OAXACA, CUICATLÁN, Colonia el progreso o Rancho Grande, Ramírez-M. et al. 127 (IBUG*); OAXACA, L. Scheinvar 3269 (MEXU); SAN JUAN BAUTISTA ATATLAUCA, Dto. De Etla., M. Vázquez 14 (MEXU); SAN JUAN BAUTISTA TUXTEPEC, Ejido Sebastopol, Ramírez-M. y Cruz-H.s.n. (UACH); SAN JUAN BAUTISTA VALLE NACIONAL, San Mateo Yetla, Ramírez-M. y Cruz-H.s.n. (UACH); SAN JOSÉ CHILTEPEC, Carretera de San Mateo Yetla a Tuxtepec, Ramírez-M. et al. 156 (IBUG*); SAN MIGUEL DEL PUERTO, La Merced del Potrero, Ramírez-M. et al. 147 (IBUG*); SANTA MARIA ALBARRADAS, San Bartolo Barrados, Ramírez-M. et al. 154 (IBUG*); SANTA MARÍA ZOQUITLÁN, Ramírez-M. et al. 152 (IBUG*); La Merced del Potrero, Ramírez-M. y Cruz-H. s.n. (UACH); Mano de León, Ramírez-M. y Cruz-H.s.n. (UACH); SANTIAGO NILTEPEC, Ramírez-M. y Cruz-H.s.n. (UACH); SANTO DOMINGO ZANATEPEC, Ejido General Fuentes, Ramírez-M. et al. 139 (IBUG*); YAUTEPEC, Lachixova, Ramírez-M. et al. 135 (IBUG*); PUEBLA, COXCATLÁN, Coxcatlán, saliendo del pueblo hacia Teotitlán, Gómez-H. 2034 (MEXU); SAN JOSÉ MIAHUATLÁN, San Mateo Tlacoxcalco, Ramírez-M. et al. 125 (IBUG*); TEHUACÁN, Bravo-Hollis 2340 (MEXU); San Pablo Tepetzingo, S. Arias 852 (MEXU); Atepeji, L. Scheinvar et al. 2616 (MEXU); Tepexi de Rodríguez, Loma atrás de San Antonio Huejonapan, G. González y R. Cid s.n. (MEXU); San Diego Chalma, L. Guzmán 849 (MEXU);San Pablo Tepoteingo, R. Castillo 579 (XAL); ZAPOTITLÁN, Zapotitlan de Salinas, T. Hernández 36 (MEXU); QUERÉTARO, CADEREYTA, Vizarrón de Montes, F. Limón 11 (MEXU); COLÓN, Colonia San Martín, L. Guzmán 88A (MEXU); JALPAN DE SERRA, El Trapíche, al norte de Jalpan, R. Rodríguez y L. Scheinvar 4606 (MEXU); Alrededores de Jalpa de Serra, L. Scheinvar 5901 (MEXU);QUERÉTARO, Al S del poblado de Hércules, en la calle contigua a la vía férrea, H. Hernández et al. 2467 (MEXU); Puerto de Ánimas, B. Rosales y O. Guerrero 2 (MEXU); QUINTANA ROO, Chetumal, km 21 carretera Chetumal-Escárcega. Ejido Juan Sarabia, L. Scheinvar 6420 (MEXU); TABASCO, NACAJUCA, Tucta, a $2 \mathrm{~km}$ de la escuela, dentro de los camellones chontales, J. Calzada 4899 (XAL); VERACRUZ, ACULTZINGO, Acultzingo, Ramírez-M. y Cruz-H.18 (UACH); COATEPEC, Barranca de Tlacuitlapa, entre Tuzamapa y Jalcomulco, G. Castillo 725 (XAL); Cerro Achichuca, ejido Tuzamapan, A. Vóvides 834 (XAL). 
Apéndice 1. Continuación

CONDICION DESCONOCIDA. CHIAPAS, ARRIAGA, cerca de Arriaga, Bravo-Hollis 4815 (MEXU); IXTACOMITAN, Rancho cerca de Ixtacomitan, D. Breedlove 26494 (MEXU); ESTADO DE MÉXICO, Valle de Bravo, Valle de Bravo, Convers 1830 (MEXU); GUERRERO, CHILPANCINGO, Rincon de la Via, H. Kruse 3109 (MEXU); Rincon de la Via, H. Kruse 3049 (MEXU); JALISCO, TAPALPA, Camino a la Casa Blanca, R. Rodríguez s.n. (IBUG); MORELOS, ALPUYECA, C. Rowell 7671 (MEXU); OAXACA, HERÓICA CIUDAD DE EJUTLA DE CRESPO, Cerro de Ejutla, C. Conzatti 3937 (MEXU); SAN JUAN MIXTEPEC, Distrito Miahuatlán, E. Hunn 1090 (MEXU); SAN MARTÍN TOXPALÁN, Km 4 al Sur de San Martín Toxpalán, L. Scheinvar 3165A (MEXU); SANTA MARIA ZOQUITLÁN, Ramírez-M. et al. s.n. (IBUG*); TABASCO, TEAPA, S. Zamudio 54 (MEXU); COMALCALCO, Tecolutilla, orilla de potrero, F. Ventura 21221 (MEXU); VERACRUZ, AMATLÁN DE LOS REYES, Orilla del camino Peñuela- Facultad de Biología, H. Oliva y G. Ramón 325 (MEXU).

\section{Hylocereus aff. escuintlensis Kimnach}

SILVESTRES. CHIAPAS, MOTOZINTLA, La Verbena, García-R. et. al 1696 (IBUG*); VILLA COMALTITLÁN, Entrada a Zapaluta, García-R. et. al 1701 (IBUG*); OCOSINGO, San Bartolo, F.J. Ramírez et al. 869 (IBUG*).

CULTIVADOS. CHIAPAS, ESCUINTLA, Cintalapa, García-R. et. al 1703 (IBUG*); Ranchería Puente Santa Rita, Ramírez-M. y Cruz-H.888 (IBUG*); MAPASTEPEC, Km 172 Carretera a Arriaga-Mapastepec, García-R. et. al 1705 (IBUG*); TAPACHULA, Palmasola, García-R. et al.1697 (IBUG*); TUXTLA CHICO, Toma la Galera, García-R. et. al 1699 (IBUG*); El Trapiche, Ramírez-M. y Cruz-H.881 (IBUG*); Camino Calanzé al Trapiche, Ramírez-M. y Cruz-H.883 (IBUG*); TUZANTÁN, Rancho las Hawallanas, Ramírez-M. y Cruz-H.879 (IBUG*).

\section{Hylocereus sp.}

SILVESTRES. OAXACA, ASUNCIÓN IXTALTEPEC, Nisanda Corona-O. et al. 143 (IBUG*); La Mata, Corona-O. et al. 145 (IBUG*). CULTIVADOS. OAXACA, ASUNCIÓN IXTALTEPEC, La Mata, Corona-O. et al. 141 (IBUG*); SANTIAGO NILTEPEC, Niltepec, Corona-O. et al. 137 (IBUG*). 